\title{
Experimental, Theoretical \& Computational Sturly of Frequency Upshift of Electromagnetic Radiation Using Plasma Techniques
}

\section{ANNUAL TECHNICAL REPORT}

DOE/ER/12114--2

DE93 005092

Submitted by:

THE REGENTS OF THE UNIVERSITY OF CALIFORNIA

UNIVERSITY OF CALIFORNIA, LOS ANGELES

SCHOOL OF ENGINEERING AND APPLIED SCIENCE

LOS ANGELES

PRINCIPAL INVESTIGATOR:

C. Joshi, Professor

Electrical Engineering Department

(310) 825-7279

Period of performance: January 15, 1992 - January 14, 1993

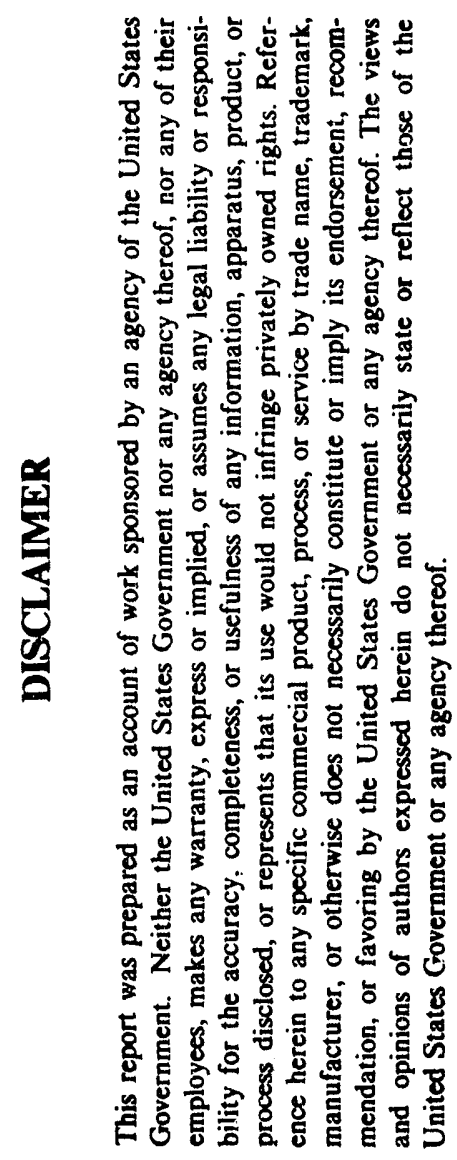

submitted to:

Dr. Walter M. Polansky

U.S. DEPARTMENT OF ENERGY

Office of Basic Energy Research

for

Grant Number DE-FG03-91-ER12114

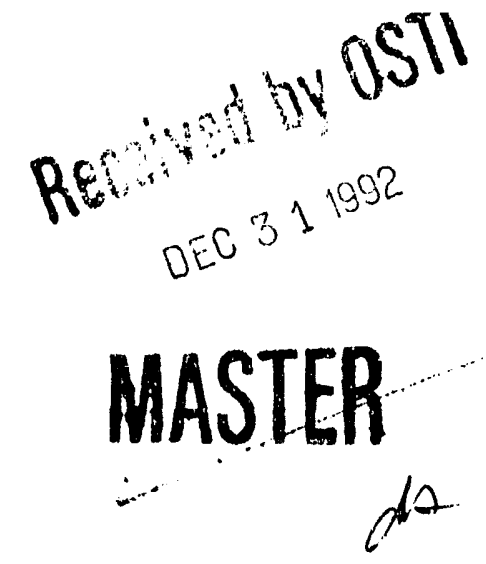

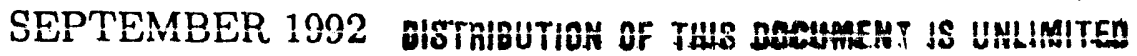




\section{Summary of Overall Progress}

This is a second year progress report on "Experimental, Theoretical and Computational Study of Frequency Upshift of Electromagnetic Radiation Using Plasma Techniques." This has been an exceptionally good year for our program. The highlights are:

I. Ionization fronts have been shown to frequency upshift e.m. radiation by greater than a factor 5 . In our experiments $33 \mathrm{GHz}$ microwave radiation is upshifted to more than 175 $\mathrm{GHz}$ using a relativistically propagating ionization front created by a laser beam.

II. A Letter describing our results has been published in Physical Review Letters and an "invited" paper has been submitted to IEEE Trans. in Plasma Science. Both papers are enclosed.

III. The IEEE Trans. in Plasma Science has decided to do a special issue on "Frequency Upconversion Using Plasma Techniques" because of the activity in this field driven by the UCLA group. Dr. Warren Mori who is a participating investigator on this grant is the Guest Editor. The issue is slated for publication in March 1993.

IV. The P.I., Co-P.I. and students have given numerous "invited talks" at prestigious conference (see list). Student Richard Savage, who was working on this program, has graduated and taken up a staff position at Caltech on the LIGO project.

V. Collaboration with Dr. Andrew Sessler (LBL) continues to go well. We had Dr. D. Kalluri of University of Massachusetts visit both UCLA and LBL during the summer. He was supported by Dr. Sessler to look at the effect of applying a magnetic field to the plasma. Preliminary indications are that, the B field gives as an independent knob to tune the frequency in the experiment. 


\section{Invited Talks}

1. Photon Frequency Upshift Using Relativistic Ionization fronts by C. Joshi, National Symposium on Science and Technology of Plasmas, Dec. 17-21, 1991, Indore, India.

2. Generation of Tunable Radiation Using a Relativistic Underdense Ionization Front by W. B. Mori, 19th International Conference on Plasma Science, June 1-3, 1992, Tampa, Florida.

3. Photon Acceleration via Laser-Produced Ionization Fronts by R. L. Savage, 8th International Conference on Ultrafast Phenomena, June 8-12, 1992, Antibes-Juan-les-pins, France.

4. Frequency Upshifting Using Underdense Ionization Fronts by W. B. Mori, International Conference on Ultra-Wideband Short-Pulse Electromagnetics, October 8-10, 1992, Brooklyn, New York. 


\section{List of Publications}

(These publications are attached to this progress report.)

1. R. L. Savage Jr., C. Joshi and W. B. Mori, "Frequency Upconversion of Electromagnetic Radiation Upon Transmission into an Ionization Front," Physical Review Letters 68, 946 (1992).

2. R. L. Savage Jr., R. P. Brogle, W. B. Mori, and C. Joshi, "Photon Acceleration Via Laser-Produced Ionization Fronts," Procecdings of the 8th International Conference on Ultrafast Phenomena, June 8-12, 1992, Antibes-Juan-les-pins, France.

3. R. L. Savage Jr., R. P. Brogle, W. B. Mori, and C. Joshi, "Frequency Upshifting and Pulse Compression via Underdense Relativistic Ionization Fronts," IEEE Trans. on Plasma Science, Special Issue on the Generation of Tunable Radiation Using Plasmas, ed. by W. Mori, March 1993.

\section{Report}

R. P. Brogle, "Reflections and the Frequency Upshift Experiment," UCLA PPG-1463, This report is the written version of the presentation given by the author for his oral qualifying examination at UCLA.

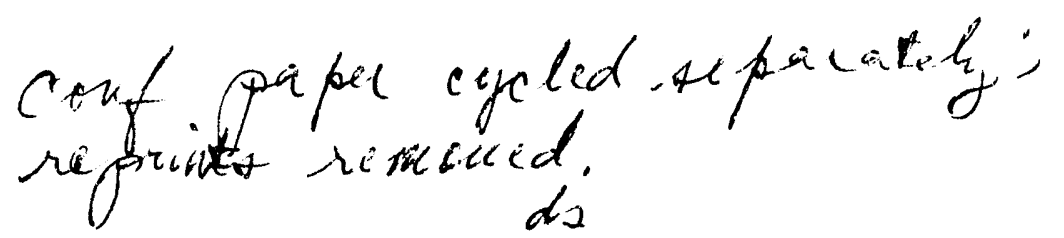




\title{
Institute of Plasma and Fusion Research
}

\author{
Reflections and the \\ Frequency Upshift Experiment \\ R. P. Brogle \\ PPG -1463 \\ September 1992
}

Affiliated with the Departments of:

Astronomy

Earth and Space Sciences

Electrical Engineering

Materials Science and Engineering

Mechanical, Aerospace and Nuclear Engineering

Physics

and

Center for Advanced Accelerators

Institute of Geophysics and Planetary Physics 


\title{
Reflections and the Frequency Upshift Experiment
}

\author{
R. P. Brogle \\ Departments of Physics and Electrical Engineering, UCLA
}

26 August 1992

\begin{abstract}
When an electromagnetic wave encounters a stationary boundary between two different media, we will observe the familiar partial reflection and partial transmission of the wave. If the boundary is moving, however, additional effects will arise including frequency shifting, pulse compression, and transmitted wave turnaround. The frequency upshift experiment at UCLA studies these effects by investigating the interaction of electromagnetic waves with a rapidly moving plasma/neutral gas boundary.

In this paper, we will first discuss some basic laws of reflection and transmission of electromagnetic waves. We will then look at the upshift experiment and give some of the results to date. Finally, we will discuss whether or not the transmitted wave turnaround predicted by the theory constitutes a new type of electromagnetic wave reflection.
\end{abstract}

This report is the writien version of the presentation given by the author for his oral qualifying examination at UCLA.

This work is supported by DOE grant number DE-FG03-91-ER12114. 


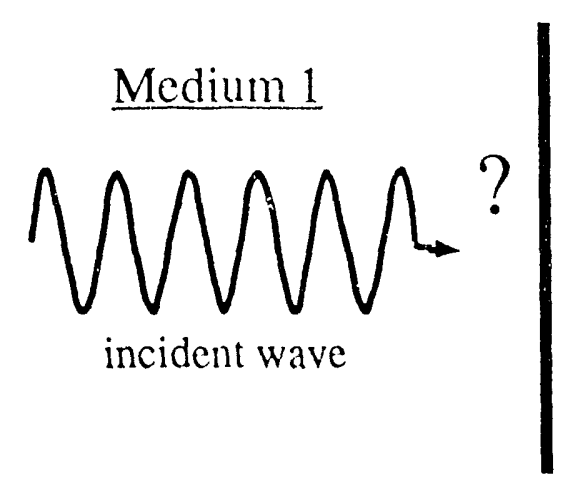

Medium 2

Figure 1: Electromagnetic wave boundary problem.

\section{Introduction}

To begin, let us familiarize ourselves with a general procedure for answering the following question: what happens when an electromagnetic wave encounters a boundary betwcen two media? (Fig. 1). To solve this problem, we will carry out the following two steps:

1. Find the possible electromagnetic modes on each side of the boundary. Each mode gives rise to a separate dispersion relation between the frequency of the wave $\omega$ and its wave number $k$. These dispersion relations are found by solving the set of electromagnetic equations which describes each medium for $\omega$ and $k$. Two of these will be the Maxwell curl equations:

$$
\begin{gathered}
\nabla \times \mathrm{E}=-\frac{1}{c} \frac{d \mathrm{~B}}{d t} \\
\nabla \times \mathrm{H}=\frac{1}{c} \frac{d \mathbf{D}}{d t}+\frac{4 \pi}{c} \mathrm{~J}
\end{gathered}
$$

and there may be additional equations to solve for the free current $J$. Once we have found the set of mathematical solutions to these equations, we must eliminate any which are physically unrealizeable. The remaining solutions for $k$ as a function of $\omega$ will then describe the possible electromagnetic modes in each medium.

2. Use the boundary conditions of the problem to solve for the electromagnetic field amplitudes of each mode. For every problem we will have the Maxwell boundary conditions:

$$
\begin{gathered}
D_{2 \perp}=D_{1 \perp}-4 \pi \sigma \\
\mathbf{E}_{2 \|}=\mathbf{E}_{1 \|} \\
B_{2 \perp}=B_{1 \perp} \\
\mathbf{H}_{2 \|}=\mathbf{H}_{1 \|}-\frac{4 \pi}{c} \mathbf{K} \times \mathbf{n}_{21}
\end{gathered}
$$

where $\sigma$ is the surface charge at the boundary, $\mathbf{K}$ is the surface current, and $\mathbf{n}_{21}$ is the unit vector perpendicular to the boundary and pointing from medium 2 into medium 1 . 
These cquations plus any additional boundary conditions will allow us to find the ratio of clectric ficld amplitude to incident electric field amplitude $\left(E / E_{0}\right)$ for each mode at the boundary surfacc.

Once we have carried out these two steps, we have effectively solved the boundary problem. We can then use the information wc've gained to determine such parameters as the power reflection and transmission coefficients $(R$ and $T)$ and the encrgy distribution of the modes.

\subsection{Approximations}

Throughout this paper, we will assume the following characteristics of our boundary problems:

1. All electromagnetic waves are plane waves of the form $e^{i(k z-\omega t)}$. With respect to our illustrations, the $+z$ direction will be to the right and the $-z$ direction will be to the left.

2. All boundaries are infinitely sharp, stationary planes.

3. The incoming radiation is at normal incidence to the boundary plane.

4. The medium on either side of the boundary is uniform and linear, having electric permittivity $\epsilon$ and magnetic permeability $\mu=1$.

With these approximations, we note that $\mathrm{D}=\epsilon \mathbf{E}, \mathbf{H}=\mathbf{B}$, the frequency of all modes is equal to the incident frequency $\omega$, and the Maxwell curl equations become:

$$
\begin{gathered}
B=\frac{c k}{\omega} E \\
\left(c^{2} k^{2}-\epsilon \omega^{2}\right) E=4 \pi i \omega J
\end{gathered}
$$

We will now do some examples of solving electromagnetic wave boundary problems.

\section{Stationary Boundaries}

\subsection{Boundary with a Dielectric}

For our first example we consider the case of an electromagnetic wave in free space encountering a boundary with a dielectric medium (Fig. 2). For a dielectric there is no free current present, thus $J=0$ and our second Maxwell curl equation (Eq. 8 ) becomes:

$$
\left(c^{2} k^{2}-\epsilon \omega^{2}\right) E=0
$$

which gives the possible modes:

$$
k= \pm \sqrt{\epsilon} \frac{\omega}{c}
$$




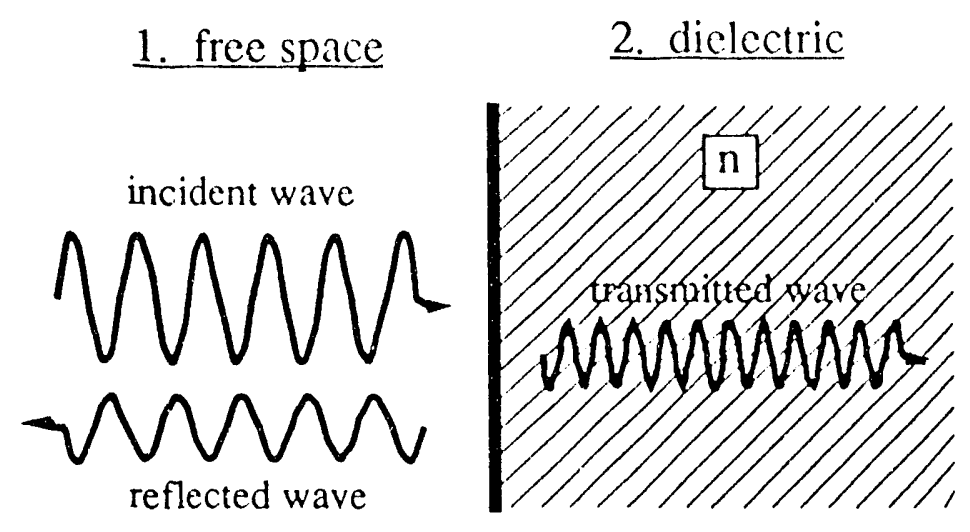

Figure 2: Electromagnetic wave encountering a boundary with a material of dielectric constant $n$.

For dielectrics, $\sqrt{\epsilon}$ is commonly referred to as the index of refraction of the material and is denoted by the symbol $n$. In free space $n=1$ and so our modes are:

$$
k= \pm \frac{\omega}{c}
$$

while in the dielectric material we have:

$$
k= \pm n \frac{\omega}{c}
$$

The solution $k=-n \omega / c$, however, indicates a wave travelling toward the boundary from the righi. Since we are assuming an incident wave from the left only, we can eliminate this mode as unphysical. Thus our problem consists of three modes: an incident wave $\left(k_{0}=+\omega / c\right)$, a reflected wave $\left(k_{r}=-\omega / c\right)$, and a transmitted wave $\left(k_{t}=+n \omega / c\right)$.

We now use the Maxwell boundary conditions to find the electromagnetic field amplitudes of each mode. For a plane wave at normal incidence to a boundary, $E_{\perp}$ and $B_{\perp}$ are zero. Also, for our dielectric there is no surface current $(\mathbf{K}=0)$ and we are assuming no free charge has been added $(\sigma=0)$. Our boundary conditions are then:

$$
\begin{aligned}
& E_{1}=E_{2} \\
& B_{1}=B_{2}
\end{aligned}
$$

In terms of the fields of the different modes in each medium these equations are:

$$
\begin{aligned}
& E_{0}+E_{r}=E_{t} \\
& B_{0}+B_{r}=B_{t}
\end{aligned}
$$

Using the first Maxwell curl equation (Eq. 7), Fq. 16 becomes:

$$
\frac{c k_{0}}{\omega} E_{0}+\frac{c k_{r}}{\omega} E_{r}=\frac{c k_{t}}{\omega} E_{t}
$$


Substituting the values of the different $k$ 's into Eq. 17 we obtain:

$$
\begin{aligned}
& E_{0}+E_{r}=E_{t} \\
& E_{0}-E_{r}=n E_{t}
\end{aligned}
$$

Solving this set of two equations for $E_{r}$ and $E_{t}$ gives:

$$
\begin{aligned}
& \frac{E_{r}}{E_{0}}=\frac{1-n}{1+n} \\
& \frac{E_{t}}{E_{0}}=\frac{2}{1+n}
\end{aligned}
$$

We now have the electric field amplitudes of the reflected and transmitted waves with respect to the amplitude of the incident wave.

If we want to know the fraction of the incident power that is transferred into each wave, we must find the ratio of reflected and transmitted wave intensities to the incident intensity. The general expression for the intensity of an electromagnetic wave is:

$$
I=|\mathbf{S}|=\frac{c}{8 \pi}\left|\mathbf{E} \times \mathbf{H}^{*}\right|
$$

where $\mathbf{S}$ is the Poynting vector of the wave. In the framework of our approximations, this expression becornes:

$$
I=\frac{c^{2} k}{8 \pi \omega}|E|^{2}
$$

Substituting the appropriate values of $k$ for the reflected and transmitted waves we can find the power reflection and transmission coefficients:

$$
\begin{gathered}
R \equiv \frac{I_{r}}{I_{0}}=\left|\frac{1-n}{1+n}\right|^{2} \\
T \equiv \frac{I_{t}}{I_{0}}=n\left|\frac{2}{1+n}\right|^{2}
\end{gathered}
$$

In order to conserve energy, the rate of energy flowing into the boundary must equal the rate of energy flowing out. Therefore, these coefficients must satisfy the condition $R+T=1$. This is easily verified from the above equations.

\subsection{Boundary with a Conductor}

We now look at the case of an electromagnetic wav's in free space encountering a boundary with a conducting medium (Fig. 3). Unlike the dielectric, there may be currents in the conductor caused by free charges responding to the electromagnetic ficlds of the wave. We assume that the conductor is ohmic, and so the current response is given by $\mathbf{J}=\sigma \mathbf{E}$, where $\sigma$ is the conductivity of the material. In the conductor Eq. 8 then becomes:

$$
\left(c^{2} k^{2}-\epsilon \omega^{2}\right) E=4 \pi i \omega \sigma E
$$




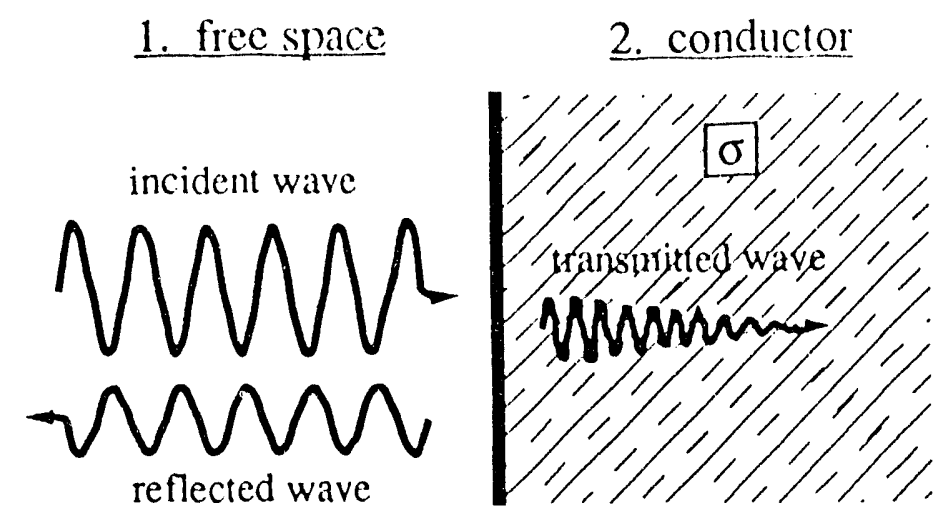

Figure 3: Electromagnetic wave encountering a boundary of conductivity $\sigma$.

The dispersion relation for the wave transmitted into the conductor is thus:

$$
c^{2} k_{t}^{2}=\epsilon \omega^{2}+4 \pi i \omega \sigma
$$

Note that $k_{t}$ is now a complex number. Solving for $k_{t}$ will give us two possible transmitted modes, one in which the wave is exponentially damped and one in which the wave is exponentially growing. Because the exponentially growing wave requires a large energy source which is not present in our simple problem, we rule it out as being unphysical. Therefore we are left with a single transmitted wave which is exponentially damped as it travels through the conductor.

In free space Eq. 8 gives us the same incident and reflected modes as before:

$$
\begin{aligned}
& k_{0}=+\frac{\omega}{c} \\
& k_{r}=-\frac{\omega}{c}
\end{aligned}
$$

Because the boundary surface is conducting, the Maxwell boundary conditions are:

$$
\begin{gathered}
E_{2}=E_{1} \\
\mathrm{~B}_{2}=\mathrm{B}_{1}-\frac{4 \pi}{c} \mathrm{~K} \times \mathrm{n}_{21}
\end{gathered}
$$

where we have allowed for the possibility of a surface current $K$. The presence of a surface current, however, implies an infinite current density $\mathbf{J}$ at the boundary. This in turn would require an infinite electric field (because $\mathbf{J}=\sigma \mathbf{E}$ ). Obviously, we do not have an infinite electric field present and so $\mathbf{K}$ must be sero for an ohmic conductor. Thus our boundary conditions are the same as for a dielectric medium:

$$
\begin{aligned}
& E_{0}+E_{r}=E_{t} \\
& B_{0}+B_{r}=B_{t}
\end{aligned}
$$


Solving these cquations as before gives:

$$
\begin{aligned}
& \frac{E_{r}}{E_{0}}=\frac{1-\tilde{n}}{1+\tilde{n}} \\
& \frac{E_{t}}{E_{0}}=\frac{2}{1+\tilde{n}}
\end{aligned}
$$

where $\tilde{n} \equiv c k_{t} / \omega$. These results look very similar to the results for a dielectric, but note that the effective index of refraction $\tilde{n}$ is now a complex number. For a perfect conductor, $\sigma$ becomes infinite and therefore $\tilde{n}$ (through $k_{t}$ ) becomes infinite. In this case we have:

$$
\begin{gathered}
\frac{E_{r}}{E_{0}}=-1 \\
\frac{E_{t}}{E_{0}}=0
\end{gathered}
$$

Thus there is total reflection, with the icisuted wave electric field inverted with respect to the incident wave clectric field.

\subsection{Boundary with a Plasina}

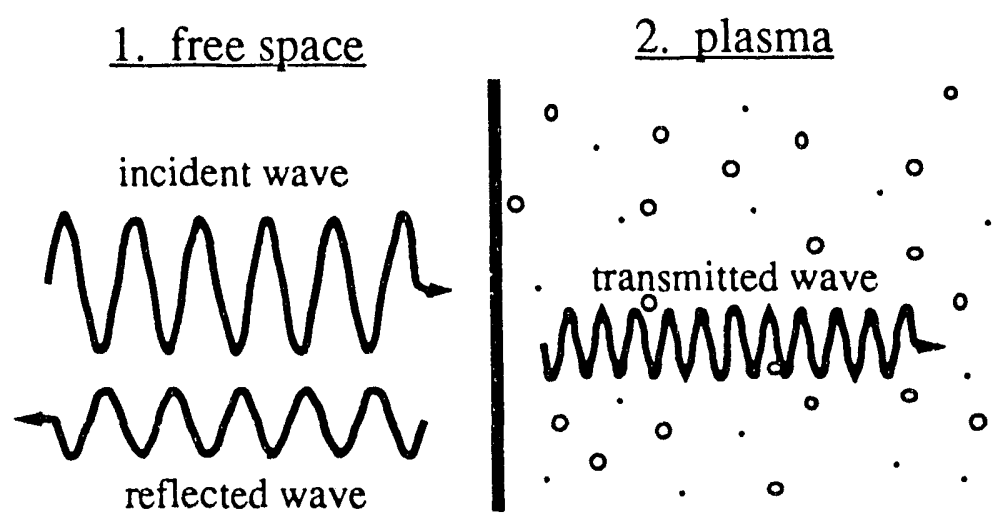

Figure 4: Electromagnetic wave encountering a plasma boundary.

Let us now study the problem of an electromagnetic wave incident on a boundary between free space and a plasma (Fig. 4). We will assume that the plasma is cold, collisionless, and has fixed ions. Because electrons in a plasma are free to move, the electromagetic wave will induce a current in the plasma. This current is given by $\mathrm{J}=-n e \mathrm{v}$, where $n$ is the electron density, $e$ is the absolute value of the electron charge, and $\mathbf{v}$ is the electron velocity in the plasma. Eq. 8 then becomes:

$$
\left(c^{2} k^{2}-\epsilon \omega^{2}\right) E=-4 \pi i \omega n e v
$$


The velocity of the plasma electrons is given by the Lorentz force law:

$$
\frac{d \mathbf{v}}{d t}=-\frac{c}{m}\left(\mathrm{E}+\frac{\mathbf{v}}{c} \times \mathrm{B}\right)
$$

Because our elcctromagnetic waves are planc waves of the form $e^{i\left(k_{2}-\omega t\right)}$ this becomes:

$$
v=-\frac{i e E}{m \omega}
$$

Here we have also assumed that the electron velocity is small, so the magnetic force can be neglected. Eq. 38 can then be written as:

$$
\left(c^{2} k^{2}-\epsilon \omega^{2}\right) E=-\frac{4 \pi e^{2} n}{m} E
$$

Because a plasma is generally dilute in comparison to a solid, the dielectric constant $\epsilon \approx 1$, and thus our dispersion relation is:

$$
c^{2} k^{2}=\omega^{2}-\omega_{p}^{2}
$$

where $\omega_{p}$ is called the plasma frequency and is given by:

$$
\omega_{p}=\left(\frac{4 \pi e^{2} n}{m}\right)^{\frac{1}{2}}
$$

This formula can be numerically approximated by $\omega_{p} \approx 2 \pi \times 9000 \sqrt{n}$, where $n$ is the plasma density in $\mathrm{cm}^{-3}$. Once again we have one physically possible transmitted mode:

$$
k_{t}=+\frac{\left(\omega^{2}-\omega_{p}^{2}\right)^{\frac{1}{2}}}{c}
$$

along with our usual free space incident and reflected modes:

$$
\begin{aligned}
& k_{0}=+\frac{\omega}{c} \\
& k_{T}=-\frac{\omega}{c}
\end{aligned}
$$

As in the case of the conducting boundary, we cannot have a surface current (because that would require an infinite electric field) and so our boundary conditions are as before:

$$
\begin{aligned}
& E_{0}+E_{r}=E_{t} \\
& B_{0}+B_{r}=B_{t}
\end{aligned}
$$

which lead to the familiar form for the electric field ratios:

$$
\frac{E_{\tau}}{E_{0}}=\frac{1-\tilde{n}}{1+\tilde{n}}
$$




$$
\frac{E_{t}}{E_{0}}=\frac{2}{1+\tilde{n}}
$$

where in this case $\tilde{n} \equiv c k_{t} / \omega=\left(1-\omega_{p}^{2} / \omega^{2}\right)^{\frac{1}{2}}$. Note that when $\omega_{p} \leq \omega, \tilde{n}$ is purely real, and when $\omega_{p}>\omega, \tilde{n}$ is purcly imaginary. Thus the power reflection coefficient, $R \equiv I_{r} / I_{0}=$ $\left|E_{r} / E_{0}\right|^{2}$, depends on the ratio $\omega_{p} / \omega$ as shown in Fig. 5 . If the plasma is underdense (that is, $\left.\omega_{p} \ll \omega\right), R \approx 0$ and we have near total transmission of the wave. If the plasma is overdense $\left(\omega_{p} \geq \omega\right)$, then $R=1$ and the wave is totally reflected. Fig. 6 shows the dependence of $R$ on plasma density $n$ for the case of incoming $35 \mathrm{GH} z$ microwaves encountering a plasma boundary. We can see that on a logarithmic scale the transition from total trans total reflection is very sudden, occurring here at a density of around $1 \times 10^{13} \mathrm{~cm}^{-3}$.

\section{Moving Boundaries}

After examining how to solve various stationary boundary problems we now $a_{i:}$ ine question: what happens if the boundary is moving at a uniform velocity? Clearly our calculations will be much more difficult because $\omega$ will change across the boundary and the simple forms of the Maxwell curl equations we have been using (Eqs. 7 and 8) will no longer be valid. However, we can get around these difficulties by performing the following steps:

1. Lorentz transform to the inertial frame in which the boundary is at rest.

2. Solve the problem for a stationary boundary as before.

3. Lorentz transform the results back to the original (laboratory) frame.

We will now do some examples of solving moving boundary problems using this method.

\subsection{Moving Perfect Conductor (Mirror)}

For our first example, we will look at the classic problem of a moving mirror approaching an electromagnetic wave in free space (Fig. 7). If the mirror is travelling at speed $\beta(\beta=v / c)$, then upon Lorentz transforming to the mirror's rest frame the incident wave frequency and wave number will be transformed as follows:

$$
\left(\begin{array}{c}
\omega_{0}^{\prime} \\
c k_{0}^{\prime}
\end{array}\right)=\left(\begin{array}{cc}
\gamma & \gamma \beta \\
\gamma \beta & \gamma
\end{array}\right)\left(\begin{array}{c}
\omega_{0} \\
c k_{0}
\end{array}\right)
$$

where, as usual, $\gamma=\left(1-\beta^{2}\right)^{-\frac{1}{2}}$. Thus in the mirror's frame (the primed frame) the frequency and wave number of the incident wave are:

$$
\begin{aligned}
& \omega_{0}^{\prime}=\gamma(1+\beta) \omega_{0} \\
& k_{0}{ }^{\prime}=\gamma(1+\beta) k_{0}
\end{aligned}
$$




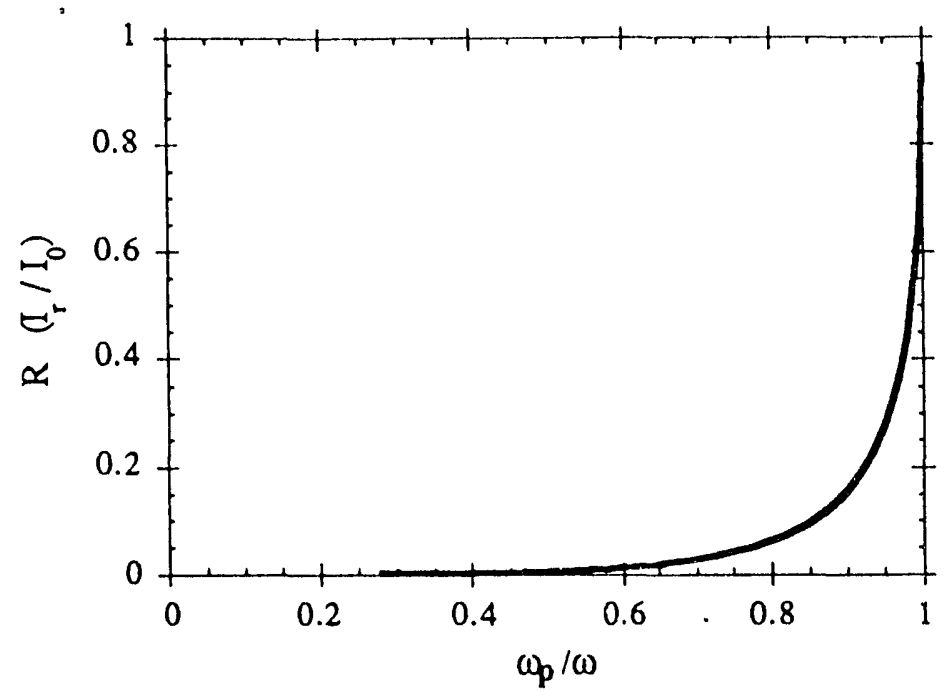

Figure 5: Reflection coefficient $R$ as a function of $\omega_{p} / \omega$.

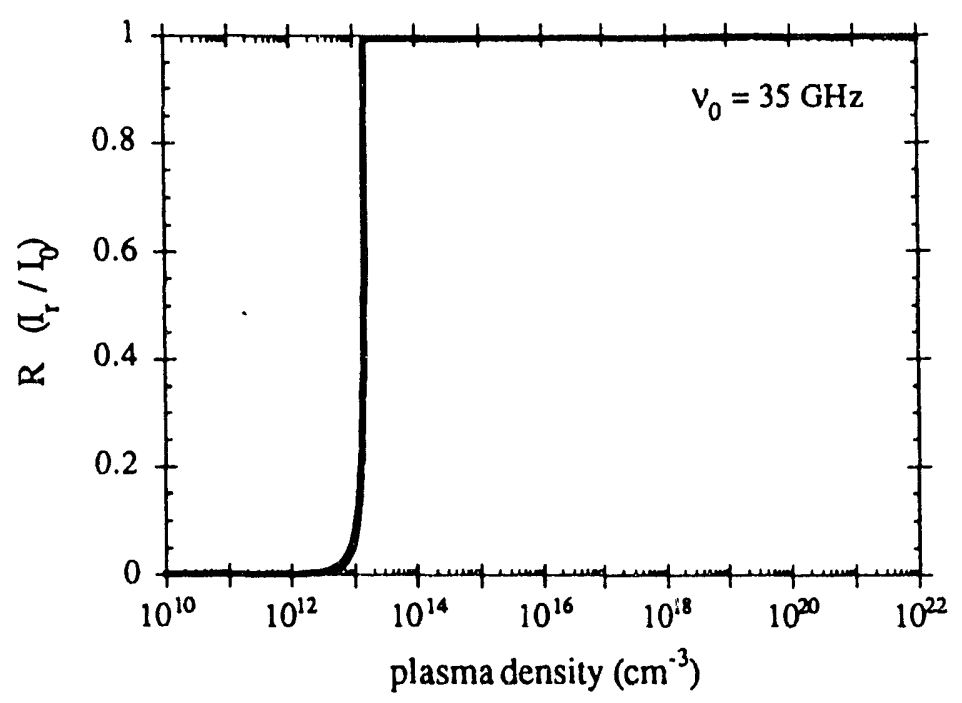

Figure 6: Reflection coefficient $R$ as a function of plasma density for an incident radiation frequency of $35 \mathrm{GHz}$. 


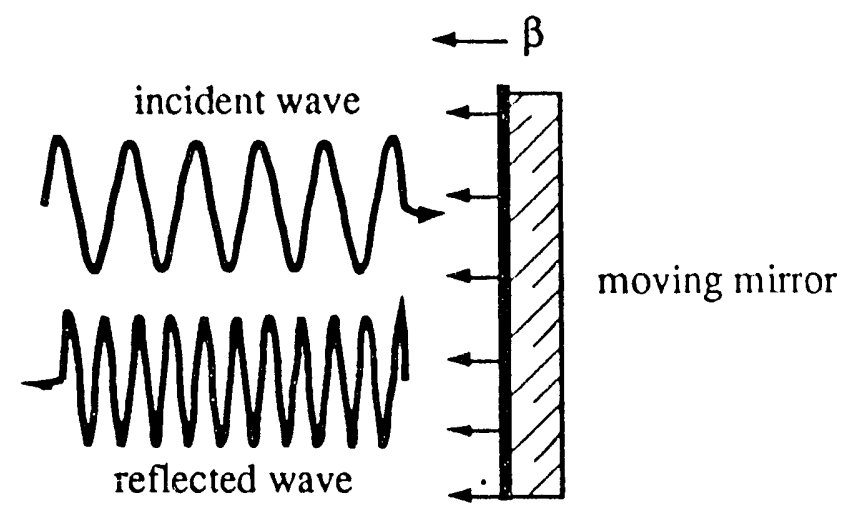

Figure 7: Electromagnetic wave encountering a moving mirror.

In this frame the wave is reflected from a stationary boundary:

$$
\begin{gathered}
\omega_{r}{ }^{\prime}=\omega_{0}{ }^{\prime} \\
k_{r}{ }^{\prime}=-k_{0}{ }^{\prime}
\end{gathered}
$$

We now perform an inverse Lorentz transformation to get back to the lab frame:

$$
\left(\begin{array}{c}
\omega_{r} \\
c k_{r}
\end{array}\right)=\left(\begin{array}{cc}
\gamma & -\gamma \beta \\
-\gamma \beta & \gamma
\end{array}\right)\left(\begin{array}{c}
\omega_{r}{ }^{\prime} \\
c k_{r}{ }^{\prime}
\end{array}\right)
$$

In terms of the incident wave frequency and wave number we find for the reflected wave:

$$
\begin{aligned}
& \omega_{r}=\gamma^{2}(1+\beta)^{2} \omega_{0} \\
& k_{r}=-\gamma^{2}(1+\beta)^{2} k_{0}
\end{aligned}
$$

Although there is no frequency change in the rest frame of the mirror (it is a reflection from a stationary boundary in this frame), we observe a large frequency upshift in the laboratory frame for significant values of $\gamma$. This, of course, is the well known Doppler effect. We note that the moving mirror carries kinetic energy in the laboratory frame which is transferred to the electromagnetic wave during the reflection. Quantum mechanically, each photon which makes up our classical wave has energy $\hbar \omega$. The energy transfer from the mirror to the wave is then characterized by the increased frequency of each reflected photon, with the total number of photons being conserved. Of course, it would be very difficult to accelerate a physical mirror to relativistic speeds; thus it is unlikely that significant frequency upshifts can be attained using this method.

\subsection{Moving Dielectric}

We now look at what happens when an electromagnetic wave in free space encounters a moving dielectric medium (Fig. 8). Employing our standard procedure for a moving boundary problem, we find that there is one reflected and one transmitted mode, just as in the 


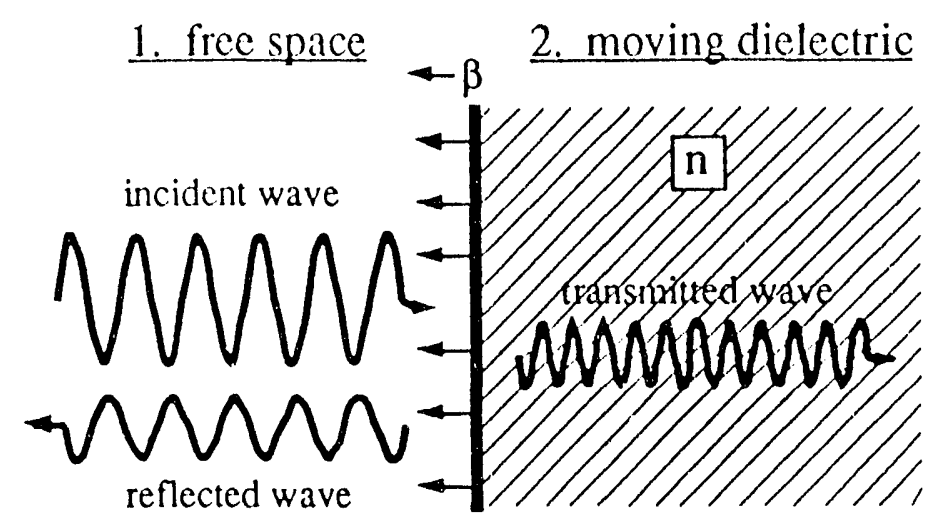

Figure 8: Electromagnetic wave encountering a moving dielectric

stationary dielectric case. The frequency and wave number for the reflected wave in the lab frame are the same as in the case of the moving mirror:

$$
\begin{aligned}
& \omega_{r}=\gamma^{2}(1+\beta)^{2} \omega_{0} \\
& k_{\tau}=-\gamma^{2}(1+\beta)^{2} k_{0}
\end{aligned}
$$

The results for the transmitted wave, however, are a bit more interesting, so let us calculate the electromagnetic fields and the Poynting vector of this wave in addition to its frequency and wave number:

$$
\begin{gathered}
\omega_{t}=\frac{1-n \beta}{1-\beta} \omega_{0} \\
k_{t}=\frac{n-\beta}{1-\beta} k_{0} \\
\mathbf{E}_{t}=\frac{2(1-n \beta)}{(1-\beta)(1+n)} \mathbf{E}_{0} \\
\mathbf{B}_{t}=\frac{2(n-\beta)}{(1-\beta)(1+n)} \mathbf{B}_{0} \\
\mathrm{~S}_{t}=\frac{c(1-n \beta)(n-\beta)}{2 \pi(1-\beta)^{2}(1+n)^{2}}\left|E_{0}\right|^{2} \hat{\mathbf{z}}
\end{gathered}
$$

Note that if $n \beta<1$ we observe a transmitted electromagnetic wave travelling to the right through the moving dielectric. However, if $n \beta=1$ the frequency and electric field become zero; therefore, we see a static, sinusoidal magnetic field in the dielectric medium. If $n \beta>1$ the Poynting vector changes sign which indicates that the transmitted wave is now travelling to the left - thus the wave has turned around. Of course, in the rest frame of the dielectric we never observe such a turnaround; the transmitted wave simply continues to travel to the right at the reduced speed $c / n$.

Physically, what is happening in the laboratory frame is the wave is being carried to the left by the moving dielectric faster than it can propagate through the dielectric to the 
right. (Because a moving dielectric has momentum and kinetic encrgy, it will carry along an electromagnetic wave in such a manner.) Thus the net result is that in the lab frame, the wave is propagating to the left. In order for $n \beta>1$, however, we need either a very high index of refraction or a very large (relativistic) velocity for the diclectric. Therefore, this effect would be difficult (although perhaps not impossible) to observe in a laboratory.

We should also note that if our moving dielectric slab has a finite length, then the transmit 'd radiation that exits the rear boundary of the dielectric into free space will emerge with the original frequency and wave number of the incident wave. This is easiest to see in the rest frame of the dielectric: a wave that enters the front end of a stationary dielectric has its wave number changed (by a factor of $n$ ) while inside the medium, but then its wave number returns to its original value upon exiting the back end of the dielectric into free space. The frequency of the wave is the same everywhere because all boundaries are stationary in this frame. Thus because the frequency and wave number are unchanged after passing completely through the diclectric in this frame, they must be unchanged in any reference frame. Therefore, in the lab frame, the moving back boundary of the dielectric "undoes" the frequency and wave number change produced by the moving front boundary.

\subsection{Moving Plasma}

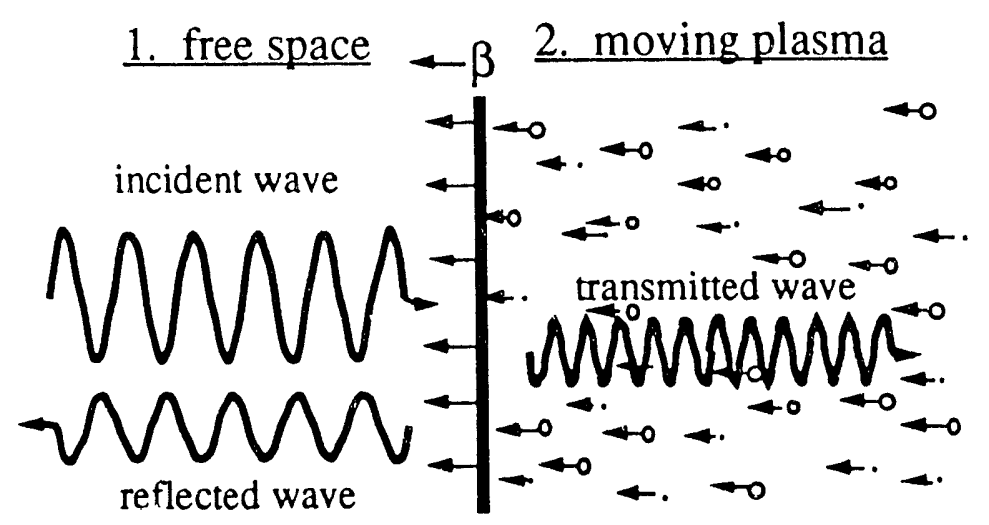

Figure 9: Electromagnetic wave encountering a moving plasma.

We now study the case of an electromagnetic wave incident on a moving plasma (Fig. 9). As before we Lorentz transform to the plasma's rest frame, perform the stationary boundary calculations, and transform back to the laboratory frame. For the reflected wave, we again obtain the moving mirror results:

$$
\begin{aligned}
& \omega_{r}=\gamma^{2}(1+\beta)^{2} \omega_{0} \\
& k_{r}=-\gamma^{2}(1+\beta)^{2} k_{0}
\end{aligned}
$$


whereas for the transmitted wave we find:

$$
\begin{aligned}
& \omega_{t}=\gamma^{2}(1+\beta)\left[1-\beta\left(1-\frac{\omega_{p}^{2}}{\omega_{0}^{2} \gamma^{2}(1+\beta)^{2}}\right)^{\frac{1}{2}}\right] \omega_{0} \\
& k_{t}=\gamma^{2}(1+\beta)\left[\left(1-\frac{\omega_{p}^{2}}{\omega_{0}^{2} \gamma^{2}(1+\beta)^{2}}\right)^{\frac{1}{2}}-\beta\right] k_{0}
\end{aligned}
$$

In Sec. 2.3 we saw that an electromagnetic wave is completcly reflected from a stationary plasma boundary when $\omega_{p} \geq \omega$ and is (nearly) completely transmitted when $\omega_{p} \ll \omega$. To determine whether an incoming wave is reflected or transmitted for a moving boundary, we must compare $\omega$ and $\omega_{p}$ in the rest frame of the plasma. In this frame, the frequency of the incoming wave is $\omega_{0}^{\prime}=\gamma(1+\beta) \omega_{0}$. Thus for a relativistically moving plasma, extremely high densities are required to make $\omega_{p} \geq \omega_{0}$ ' so that the plasma is overdense and reflects the incident radiation (Fig. 10). Therefore, relativistically moving plasmas (such a: high energy electron beams) will be in general underdense for most incident radiation frequencies.

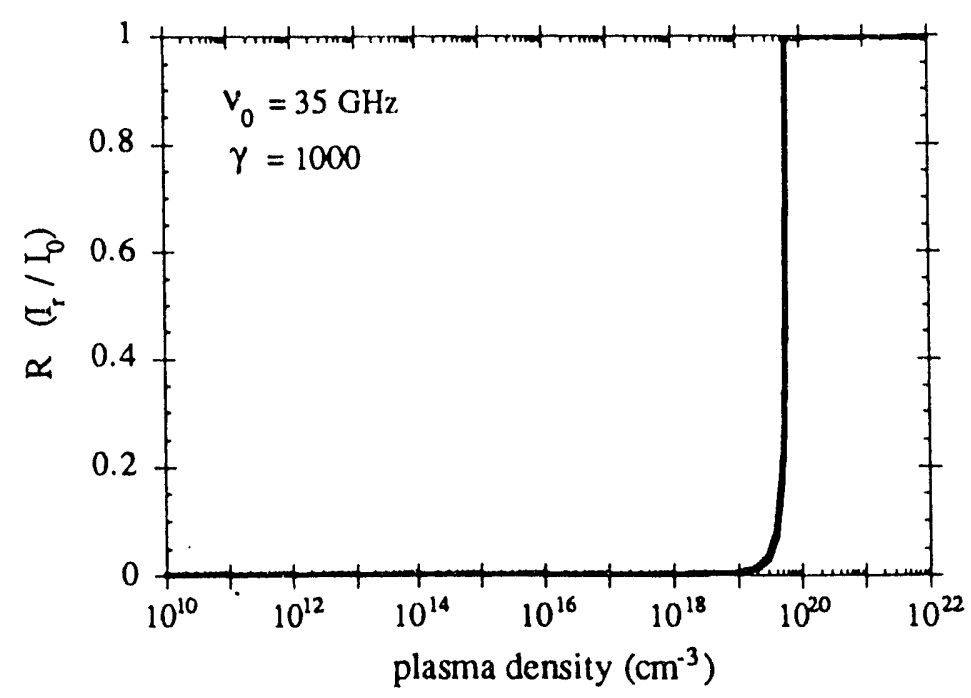

Figure 10: Reflection coefficient $R$ as a function of plasma density for incident $35 \mathrm{GHz}$ microwaves encountering a relativistically propagating plasma $(\gamma=1000)$.

For the case of a highly relativistic, underdense plasma $\left(\beta \approx 1, \omega_{p} \ll \omega_{0}^{\prime}\right)$ the transmitted wave frequency and wave number reduce to:

$$
\omega_{t}=\left(1+\frac{1}{4} \frac{\omega_{p}^{2}}{\omega_{0}^{2}}\right) \omega_{0}
$$




$$
k_{\iota}=\left(1-\frac{1}{4} \frac{\omega_{p}^{2}}{\omega_{0}^{2}}\right) k_{0}
$$

We sec that even though the wave is transmitted, we still obtain a frequency upshift which is proportional to the plasma's rest frame density $n$ (because $\omega_{p}^{2}=4 \pi e^{2} n / m$ ). Note that when $\omega_{p}^{2}=4 \omega_{0}^{2}$, the transmitted wave number $k_{t}$ is zero and for $\omega_{p}^{2}>4 \omega_{0}^{2}, k_{t}$ becomes negative. Thus when the plasma density is great enough, the transmitted wave will turn around and propagate in the opposite dircction of the incident wave. Once again, if we look in the plasma's rest frame the transmitted wave never turns around -- it simply continues through the plasma at a lower group velocity. In the lab frame, however, this transmitted wave turnaround is present above the critical value of $\omega_{p}=2 \omega_{0}$. Another interesting property of Eqs. 70 and 71 is that because the amount of upshift is proportional to $\omega_{p}^{2} / 4 \omega_{0}^{2}$, lower incident frequencies lead to higher frequency upshifts and greater changes in wave number for a given plasma density.

\subsection{Stationary Plasma, Moving Ionization Front}

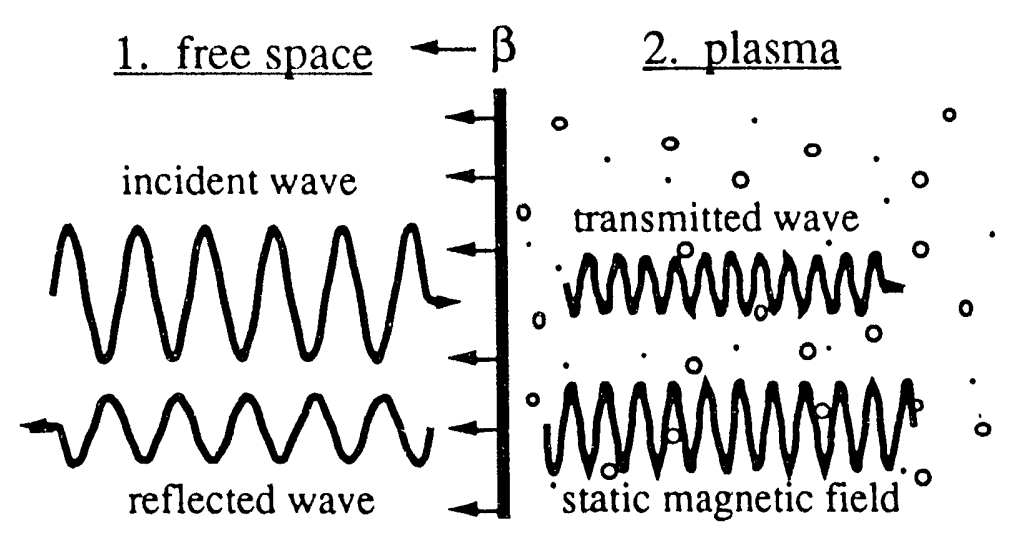

Figure 11: Electromagnetic wave encountering an ionization front moving through a stationary gas.

Our final case study is that of an electromagnetic wave encountering an ionization front moving through a stationary gas (Fig. 11) $[1,2,3]$. This is the scenario that we have been studying in the frequency upshift experiment at UCLA. Such an ionization front can be created by firing an intense laser pulse through a volume of neutral gas. This laser pulse ionizes a fraction of the gas molecules as it propagates through the volume, thus creating a rapidly moving boundary between neutral gas and plasma. We should note that this ionization front carries no energy or momentum, and unlike the case of the moving mirror the number of photons which impinge on this boundary will not be conserved. To investigate what happens when an electromagnetic wave encounters such a boundary, we transform to the rest frame of the ionization front. In this frame we see electron-ion pairs being created at the boundary and then streaming away at the front velocity $\beta$. Our Maxwell curl equations 
(Wqs. 7 and 8) in the boundary's frame (the primed frame) are:

$$
\begin{gathered}
B^{\prime}=\frac{c k^{\prime}}{\omega^{\prime}} E^{\prime} \\
\left(c^{2} k^{\prime 2}-\epsilon \omega^{\prime 2}\right) E^{\prime}=4 \pi i \omega^{\prime 2} J^{\prime}
\end{gathered}
$$

The current $J^{\prime}$ in this frame is given by:

$$
J^{\prime}=-\gamma n e v
$$

because the plasma density in this frame is greater than the plasma density in the lab frame by a factor of $\gamma$ (due to the Lorentz contraction). Now the electron velocity $v$ in this frame is found by solving the elcctron equation of motion:

$$
\frac{\partial \mathbf{v}}{\partial t}+c \beta \frac{\partial \mathbf{v}}{\partial z}=\frac{-e}{\gamma m}(\mathbf{E}+\boldsymbol{\beta} \times \mathbf{B})
$$

Assuming, as usual, solutions of the form $e^{i(k z-\omega t)}$, we solve the above equations to find the dispersion relation for electromagnetic waves in the plasma:

$$
\left(k^{\prime 2}-\frac{\omega^{\prime 2}-\omega_{p}^{2}}{c^{2}}\right)\left(k^{\prime}-\frac{\omega^{\prime}}{c \beta}\right)=0
$$

From this, we obtain two physically possible transmitted modes in the plasma (which we label with subscripts $t$ and $m$ ):

$$
\begin{gathered}
k_{t}^{\prime}=\frac{\omega^{\prime}}{c}\left(1-\frac{\omega_{p}^{2}}{\omega^{\prime 2}}\right)^{\frac{1}{2}} \\
k_{m}^{\prime}=\frac{\omega^{\prime}}{c \beta}
\end{gathered}
$$

Transforming these results back to the lab frame, we find for the first mode:

$$
\begin{gathered}
\omega_{t}=\gamma^{2}(1+\beta)\left[1-\beta\left(1-\frac{\omega_{p}^{2}}{\omega_{0}^{2} \gamma^{2}(1+\beta)^{2}}\right)^{\frac{1}{2}}\right] \omega_{0} \\
k_{t}=\gamma^{2}(1+\beta)\left[\left(1-\frac{\omega_{p}^{2}}{\omega_{0}^{2} \gamma^{2}(1+\beta)^{2}}\right)^{\frac{1}{2}}-\beta\right] k_{0}
\end{gathered}
$$

which, for a relativistic, underdense ionization front, reduce to:

$$
\omega_{t}=\left(1+\frac{1}{4} \frac{\omega_{p}^{2}}{\omega_{0}^{2}}\right) \omega_{0}
$$




$$
k_{t}=\left(1-\frac{1 \omega_{p}^{2}}{4 \omega_{0}^{2}}\right) k_{0}
$$

'Thus the results for this mode are the same as for the moving bulk plasma, with the magnitude of the frequency upshift proportional to the plasma density, and the wave turning around when $\omega_{p}^{2}>4 \omega_{0}^{2}$. (One note concerning this turnaround: as we mentioned earlier, an ionization front moving through a stationary plasma carrics no momentum and no energy. It simply creates electron-ion pairs as it travels through the neutral gas. Thus it is interesting that the transmitted wave can be turned around by the ionization front even though the front cannot impart any momentum to the wave. We'll have more to say about this phenomenon in Sec. 5.)

Transforming the second mode into the lab frame we obtain:

$$
\begin{gathered}
\omega_{m}=0 \\
k_{m}=\frac{1+\beta}{\beta} k_{0}
\end{gathered}
$$

Now from Eq. 7 we have:

$$
E_{m}=\frac{\omega_{m}}{c k_{m}} B_{m}=0
$$

and therefore the second mode is a static magnetic field in the lab frame which is sinusoidal along the $z$ direction:

$$
B_{m}(z)=B_{m} e^{i k_{m} z}
$$

The boundary conditions for this problem in the rest frame of the front are:

$$
\begin{gathered}
E_{1}{ }^{\prime}=E_{2}{ }^{\prime} \\
B_{1}{ }^{\prime}=B_{2}{ }^{\prime} \\
J_{1}{ }^{\prime}=J_{2}{ }^{\prime}=0
\end{gathered}
$$

The last boundary condition arises from the fact that when the gas molecules are first ionized, the newly-born electrons have random velocities and directions from the photo-ionization process. Thus initially there is no net current. Once the electrons stream away from the boundary, however, they begin responding to the electromagnetic fields of the incident and transmitted waves and thus create oscillating currents back in the plasma. Precisely at the boundary, though, these oscillations have not yet begun and so the current there will always be zero. Upon applying the Maxwell curl equations for the various electromagnetic modes these boundary conditions become:

$$
\begin{gathered}
E_{0}{ }^{\prime}+E_{r}{ }^{\prime}=E_{t}{ }^{\prime}+E_{m}{ }^{\prime} \\
k_{0}{ }^{\prime} E_{0}{ }^{\prime}-k_{0}{ }^{\prime} E_{r}{ }^{\prime}=k_{t}{ }^{\prime} E_{t}{ }^{\prime}+k_{m}{ }^{\prime} E_{m}{ }^{\prime} \\
k_{0}{ }^{2} E_{0}{ }^{\prime}+k_{0}{ }^{2} E_{r}{ }^{\prime}=k_{t}{ }^{2} E_{t}{ }^{\prime}+k_{m}{ }^{\prime 2} E_{m}{ }^{\prime}
\end{gathered}
$$


Solving this system of equations and then transforming the results to the laboratory frame. gives:

$$
\begin{gathered}
\frac{E_{r}}{E_{0}}=\frac{1-\tilde{n}}{1+\tilde{n}} \\
\frac{E_{t}}{E_{0}}=\frac{2}{1+\tilde{n}} \\
\frac{B_{m}}{E_{0}}=\frac{2 \beta(1-\tilde{n})}{1-\beta \tilde{n}}
\end{gathered}
$$

where $\tilde{n} \equiv c k_{t}{ }^{\prime} / \omega^{\prime}$.

Let us now calculate how much of the incident electromagnetic energy is transferred into each mode. We will do this for the following two cases:

Case 1: underdense limit, low upshift $(\tilde{n} \approx 1, \beta<1)$

In this case, the above field ratios become:

$$
\begin{aligned}
& \frac{E_{r}}{E_{0}} \approx 0 \\
& \frac{E_{t}}{E_{0}} \approx 1 \\
& \frac{B_{m}}{E_{0}} \approx 0
\end{aligned}
$$

and so nearly all of the incident energy goes into the transmitted wave mode.

Case 2: underdense limit, high upshift $(\tilde{n} \approx 1, \beta \approx 1)$

For this case we have:

$$
\begin{aligned}
& \frac{E_{r}}{E_{0}} \approx 0 \\
& \frac{E_{t}}{E_{0}} \approx 1 \\
& \frac{B_{m}}{E_{0}} \approx 2
\end{aligned}
$$

Upon first glance it appears as if energy conservation is being violated, so let us look more closely at the energy contained in the fields of each mode.

To find the total energy (per unit area) of an electromagnetic plane wave we need to multiply the energy density by the spatial length of the wave. The total energy contained in the transmitted and magnetic modes is then:

$$
\begin{gathered}
W_{t}=\frac{\left|E_{t}\right|^{2}}{8 \pi} l_{t} \\
W_{m}=\frac{\left|B_{m}\right|^{2}}{16 \pi} l_{m}
\end{gathered}
$$


We now need to find the pulselengths $l_{t}$ and $l_{m}$. To find the length of the transmitted wave, we note that because there ars no radiation sources ai the plasma boundary, the number of wave oscillations must be conserved across the ionization front. This means that:

$$
\begin{gathered}
\omega_{t} \Delta t_{t}=\omega_{0} \Delta t_{0} \\
\Delta t_{i}=\frac{\omega_{0}}{\omega_{t}} \Delta t_{0} \\
l_{t} \approx c \Delta t_{t}=c \Delta t_{0} \frac{\omega_{0}}{\omega_{t}}=l_{0} \frac{\omega_{0}}{\omega_{t}}
\end{gathered}
$$

Thus we see that the transmitted wave is compressed in length by a factor of $\omega_{0} / \omega_{t}$.

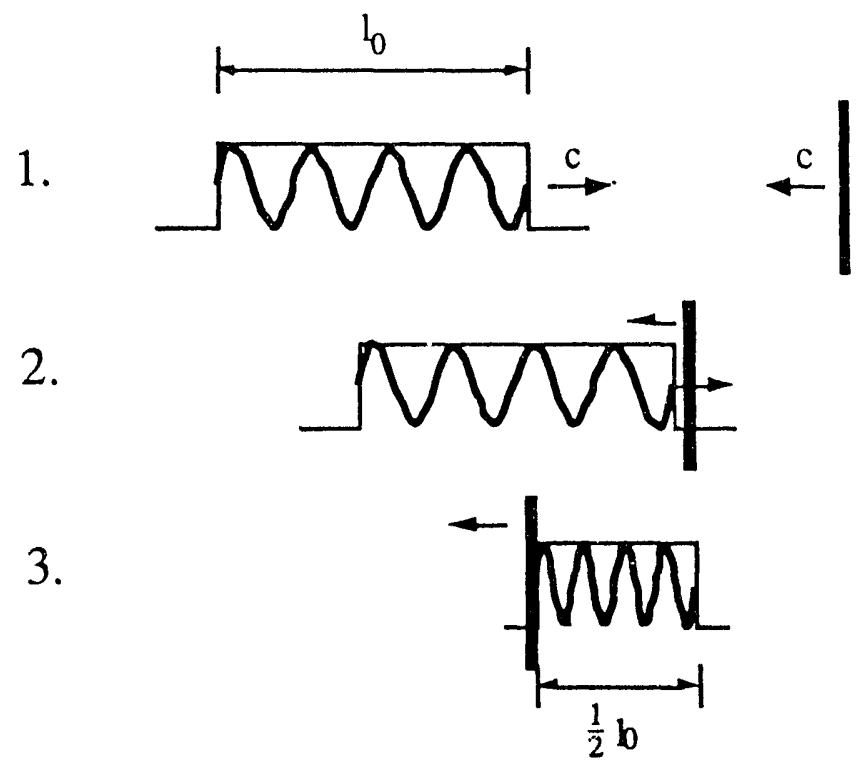

Figure 12: Illustration of the electromagnetic wave/ionization front interaction distance.

To find the length of the magnetic wave, we note that in the lab frame the incident wave pulse and the ionization front are approaching each other at the same speed $c$. The interaction distance (which is the distance over which the static magnetic field is created) is then one half of the incident wave pulselength (Fig. 12). Therefore $l_{m}=\frac{1}{2} l_{0}$. We can now calculate the total energy of the transmitted and magnetic modes:

$$
\begin{gathered}
\frac{W_{t}}{W_{0}}=\frac{\omega_{0}}{\omega_{t}} \approx 0 \\
\frac{W_{m}}{W_{0}} \approx 1
\end{gathered}
$$

Thus energy is conserved after all, and we find that for large upshifts nearly ail of the incident energy is deposited in the static magnetic field. 


\section{Experiment}

The frequency upshift experiment at UCLA was designed to investigate this last scenario: an clectromagnetic wave incident on an ionization front moving rapidly through a stationary gas [4]. The experiment was performed in a waveguide rather than in free space, and so the theory must be slightly modified to take this into account. All of the basic principles described above still apply, however, and the general behavior of the interaction process is the same. There were actually two experiments performed, a forward wave experiment and a backward wave experiment, which we now describe.

\subsection{Forward Wave Experiment}

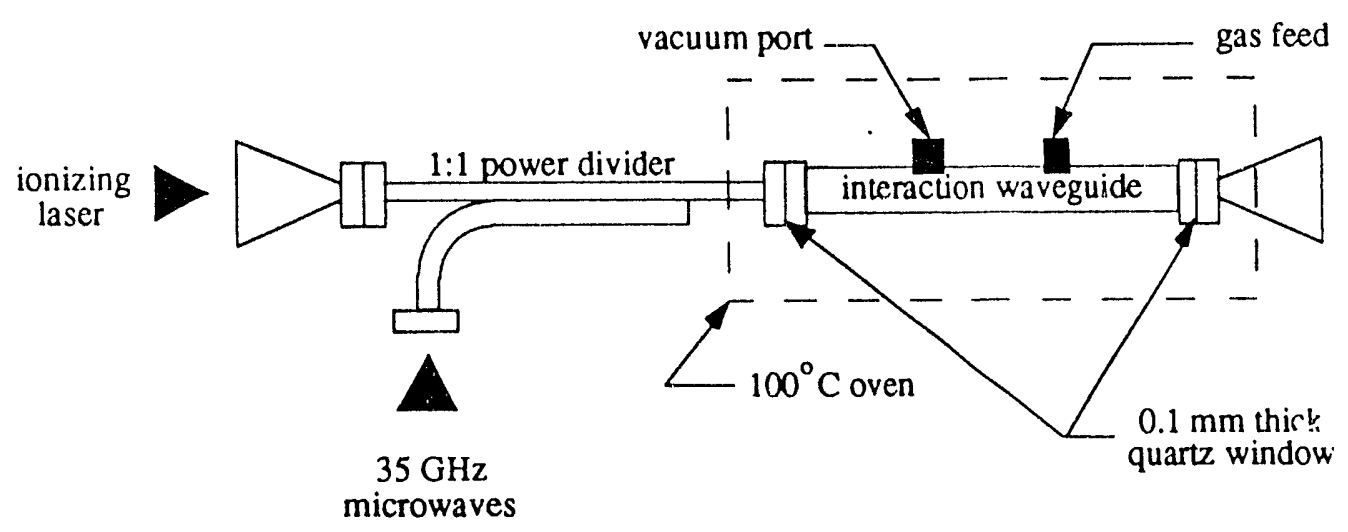

Figure 13: Forward wave experimental setup.

The setup for this experiment is shown in Fig. 13. A section of WR-19 waveguide (cutoff frequency $\nu_{c}=31.41 \mathrm{GHz}$ ) is sealed with $0.1 \mathrm{~mm}$ thick quartz windows at each end and filled with a low pressure $(0-200$ millitorr) of azulene gas. (An oven is needed to heat the azulene to $\sim 100^{\circ} \mathrm{C}$ so that these vapor pressures can be attained.) Azulene gas was chosen because it is easily ionized by an ultraviolet laser. Using a high power pulsed magnetron (10 kW instantaneous power in $300 \mathrm{~ns}$ pulses), $35 \mathrm{GHz}$ microwaves are propagated down the waveguide chamber. Because the frequency of these microwaves is close to the cutoff frequency of the waveguide, their group velocity in the guide, $v_{g}$, is only about $0.4 c$. While the microwaves are traveling through the chamber, we fire an intense ultraviolet laser pulse (wavelength $=266 \mathrm{~nm}$, energy $=40 \mathrm{~mJ}$, pulsewidth $=50 \mathrm{ps}$ ) down the chamber in the same direction as the microwaves. This creates an ionization front that travels at approximately the group velocity of the laser pulse, which is extremely close to $c$. As this ionization front overtakes the slower travelling microwaves, we look for frequency upshifts and pulse compressions of the incident $35 \mathrm{GHz}$ microwaves as predicted by the theory. 


\subsection{Backward Wave Experiment}

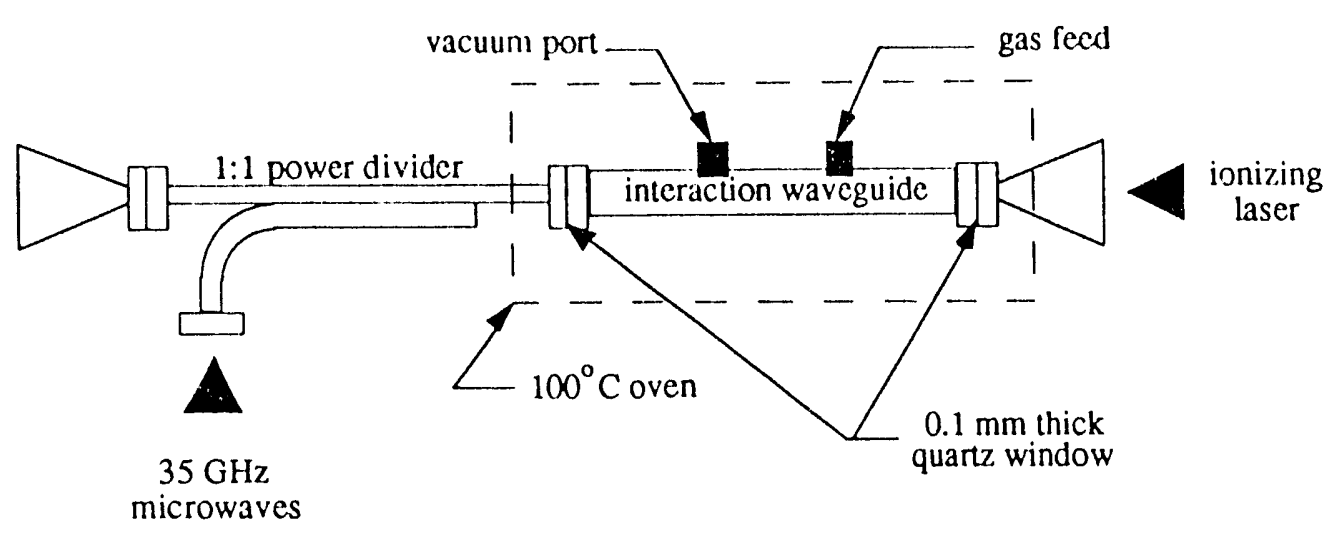

Figure 14: Backward wave experimental setup.

Our configuration for this experiment is shown in Fig. 14. For this setup we simply launch the laser in the opposite direction than in the forward wave experiment, so that the microwaves hit the iorization front head on. The theoretical predictions for this experiment are quite different than for the forward wave, and so our results should reflect these variations.

\subsection{Theoretical Predictions for the Experiment}

Following our standard procedure, we can calculate the expected frequency and wave number for the transmitted wave inside the plasma (taking the waveguide effects into account):

$$
\begin{aligned}
& \omega_{t}\left(\begin{array}{l}
f \\
b
\end{array}\right)=\omega_{0} \gamma^{2}\left(1 \mp \beta v_{g} / c\right)\left[1-\beta\left(1-\frac{\omega_{c}^{2}+\omega_{p}^{2}}{\omega_{0}^{2} \gamma^{2}\left(1 \mp \beta v_{g} / c\right)^{2}}\right)^{\frac{1}{2}}\right] \\
& k_{t}\left(\begin{array}{l}
f \\
b
\end{array}\right)=k_{0} \gamma^{2}\left(1 \mp \beta v_{g} / c\right)\left[\left(1-\frac{\omega_{c}^{2}+\omega_{p}^{2}}{\omega_{0}^{2} \gamma^{2}\left(1 \mp \beta v_{g} / c\right)^{2}}\right)^{\frac{1}{2}}-\beta\right]
\end{aligned}
$$

where the upper $f$ and lower $b$ denote the forward and backward wave results. The values of the various experimental parameters are as follows:

$$
\begin{array}{ll}
\text { incident wave frequency : } & \nu_{0}=\omega_{0} / 2 \pi=34.76 \mathrm{GHz} \\
\text { waveguide cutoff frequency : } & \nu_{c}=\omega_{c} / 2 \pi=31.41 \mathrm{GHz} \\
\text { laser frequency : } & \nu_{l}=\omega_{l} / 2 \pi=1.13 \times 10^{15} \mathrm{~Hz} \\
\text { plasma frequency : } & \nu_{p}=\omega_{p} / 2 \pi=9000 \sqrt{n} \mathrm{~Hz}\left(\text { for } n \text { given in } \mathrm{cm}^{-3}\right) \\
\text { incident microwave group velocity : } & v_{g}=c\left(1-\frac{\omega_{c}^{2}}{\omega_{0}^{2}}\right)^{\frac{1}{2}}=0.43 c \\
\text { ionization front velocity : } & \beta=\left(1-\frac{\omega_{c}^{2}+\omega_{p}^{2}}{\omega_{l}^{2}}\right)^{\frac{1}{2}} \approx 1
\end{array}
$$

Because all of our parameters are constants except the plasma frequency, the transmitted wave frequency and wave number will be a function of the ionization front plasma density 
only. We are able to set this front density simply by adjusting the ncutral gas pressure in the chamber (we determined the proportionality between the neutral gas pressure and the front density using a $65 \mathrm{Gllz}$ microwave interferometer).

Fig. 15 shows a graph of Eqs. 109 and 110 for the forward and backward wave upshifted frequency vs. front density. The highest upshifted frequency possible for both the forward and backward transmitted wave is the laser frequency, $1.13 \times 10^{15} \mathrm{~Hz}$. At densities above this point for each wave, the ionization front becomes overdense and thus the transmitted wave no longer can exist (the incident wave is completely reflected).

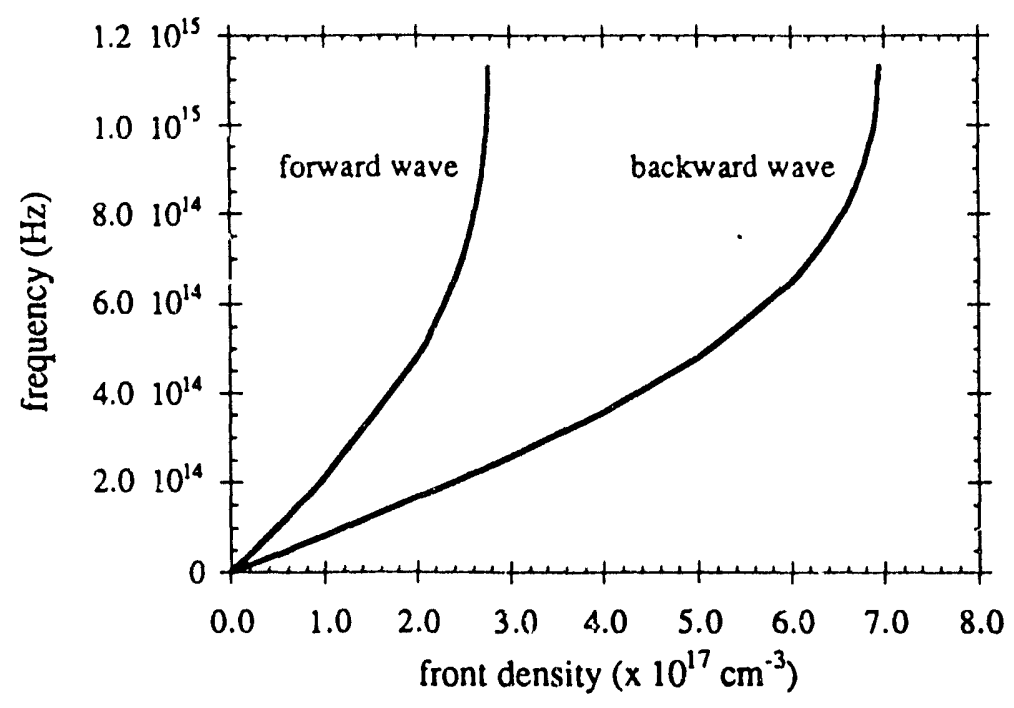

Figure 15: Theory for upshifted forward and backward wave frequencies at high ionization front densities.

For this experiment, howtver, the ionization front plasma densities are about four orders of magnitude lower than this point and so we are always operating well in the underdense regime. Thus we can use the underdense approximation $\omega_{c}^{2}+\omega_{p}^{2} \ll \omega_{0}^{2} \gamma^{2}\left(1 \mp \beta v_{g} / c\right)^{2}$. We can also assume that $\beta=1$ because our ionizing laser pulse travels at very close to $c$. With these approximations, we obtain a (slightly) simpler expression for the frequency and wave number of the transmitted wave:

$$
\begin{aligned}
& \omega_{t}\left(\begin{array}{l}
f \\
b
\end{array}\right) \approx \frac{\omega_{0}^{\prime}}{2}\left(1 \mp v_{g} / c\right)\left[1+\frac{\omega_{c}^{2}+\omega_{p}^{2}}{\omega_{0}^{2}\left(1 \mp v_{g} / c\right)^{2}}\right] \\
& k_{t}\left(\begin{array}{l}
f \\
b
\end{array}\right) \approx \frac{k_{0}}{2}\left(1 \mp v_{g} / c\right)\left[1-\frac{\omega_{c}^{2}+\omega_{p}^{2}}{\omega_{0}^{2}\left(1 \mp v_{g} / c\right)^{2}}\right]
\end{aligned}
$$

As was the case in free space, the transmitted wave frequency and wave number in the underdense limit is linearly dependent on the plasma density of the front (Fig. 16). 


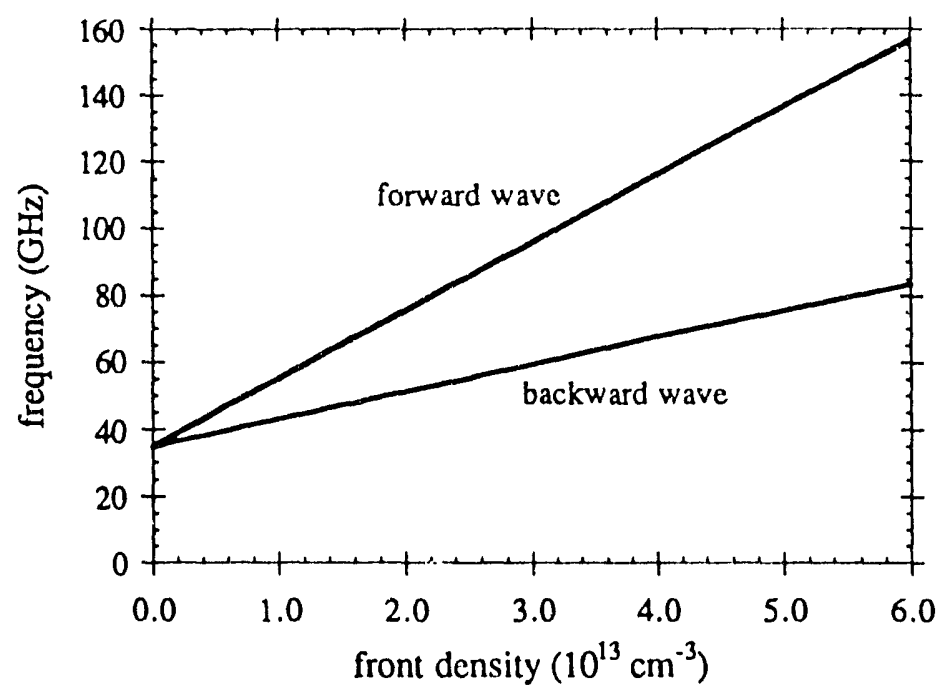

Figure 16: Theoretical prediction for upshifted forward and backward wave frequencies at low ionization front densities.

Note that the forward wave experiences a greater upshift than the backward wave. The explanation for this is as follows: From Eq. 81 we see that, in general, greater upshifts are attained for lower incident frequencies $\omega_{0}$. Now in the rest frame of the ionization front, the forward wave has a lower frequency than the backward wave. (This is apparent when we Lorentz transform both waves into the frame of the front.) This lower frequency then leads to a higher frequency upshift for the forward wave than that of the backward wave.

Physically, here's what happens inside the waveguide/plasma chamber for the forward and backward wave cases:

\section{Forward wave}

When the laser pulse enters the front window into the azulene-filled chamber, it creates and ionization front which travels down the chamber at a speed very close to $c$. As this ionization front overtakes the forward wave, it upshifts the frequency of each part of the wave that it encounters as it travels down the waveguide chamber. This upshift occurs because the wave is crossing a moving plasma/neutral boundary as described in Sec. 3.4. As each part of the forward wave is upshifted in frequency, its group velocity $(d \omega / d k)$ increases and so it follows the ionization front down the chamber at a higher speed than it had before. This increase in velocity is what causes the temporal compression of the wave. Now the laser pulse will always be the fastest object in the waveguide, and so it exits the back window of the chamber ahead of the upshifted radiation. Once the laser pulse exits, there is a boundary between plasma (inside the chamber) and neutral gas (outside the chamber) at the quartz window. This boundary is stationary in the laboratory frame. When the 
upshifted forward wave reaches the window, it exits the chamber through this stationary plasma/neutral boundary, and so its upshifted frequency is unchanged. Thus because the microwaves entered the plasma through a moving boundary but exitcd through a stationary boundary, they retain their frequency upshift. Therefore we can measure the frequency of the upshifted wave outside the chamber in free space.

\section{Backward wave}

As the incident backward wave crosses the ionization front boundary, its frequency is upshifted but for low front densities the magnitude of its group velocity decreases. As we make the front density greater, the upshifted backward wave group velocity eventually goes to zero and then changes sign (Fig. 17). Thus the transmitted backward wave is now travelling in the opposite direction of the incident wave. This is the "backward wave turnaround", which is the effect discussed in Sec. 3.4. For greater densities the backward wave's group velocity increases in this direction and we see a temporal compression as in the case of the forward wave. Once again, the incident wave enters the plasma through the moving ionization front but exits through a stationary window, and so we can measure the upshifted frequency of the backward wave in free space.

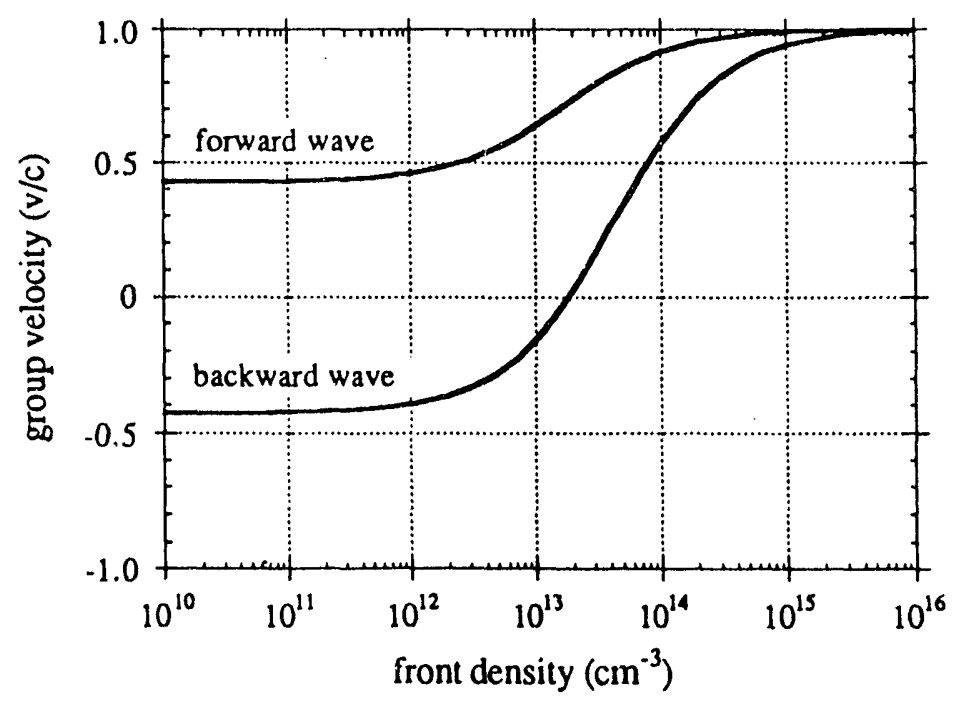

Figure 17: Group velocity of the upshifted forward and backward wave. Note the backward wave turnaround which occurs at a front density of $2 \times 10^{13} \mathrm{~cm}^{-3}$.

The prediction for the temporal pulsewidth of the forward and backward transmitted wave from the theory is given in Fig. 18. Notice that at a density of $2 \times 10^{13}$ (that is, when $\left.\omega_{c}^{2}+\omega_{p}^{2}=\omega_{0}^{2}\left(1+v_{g} / c\right)^{2}\right)$ the backward wave pulsewidth becomes infinite. This is the point where $k_{t}$ goes to zero, and thus the group velocity of the backward wave goes to zero as the backward wave turns around. At densities greater than this, both the forward and beckward wave become highly compressed. 


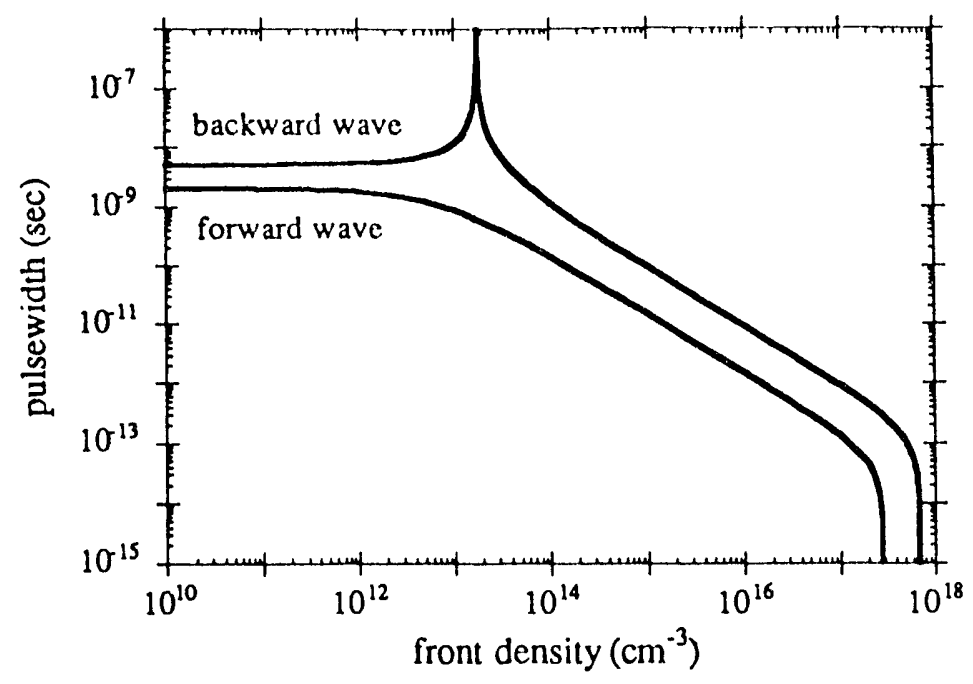

Figure 18: Theoretical prediction for upshifted forward and backward wave pulsewidths.

\subsection{Experimental Results}

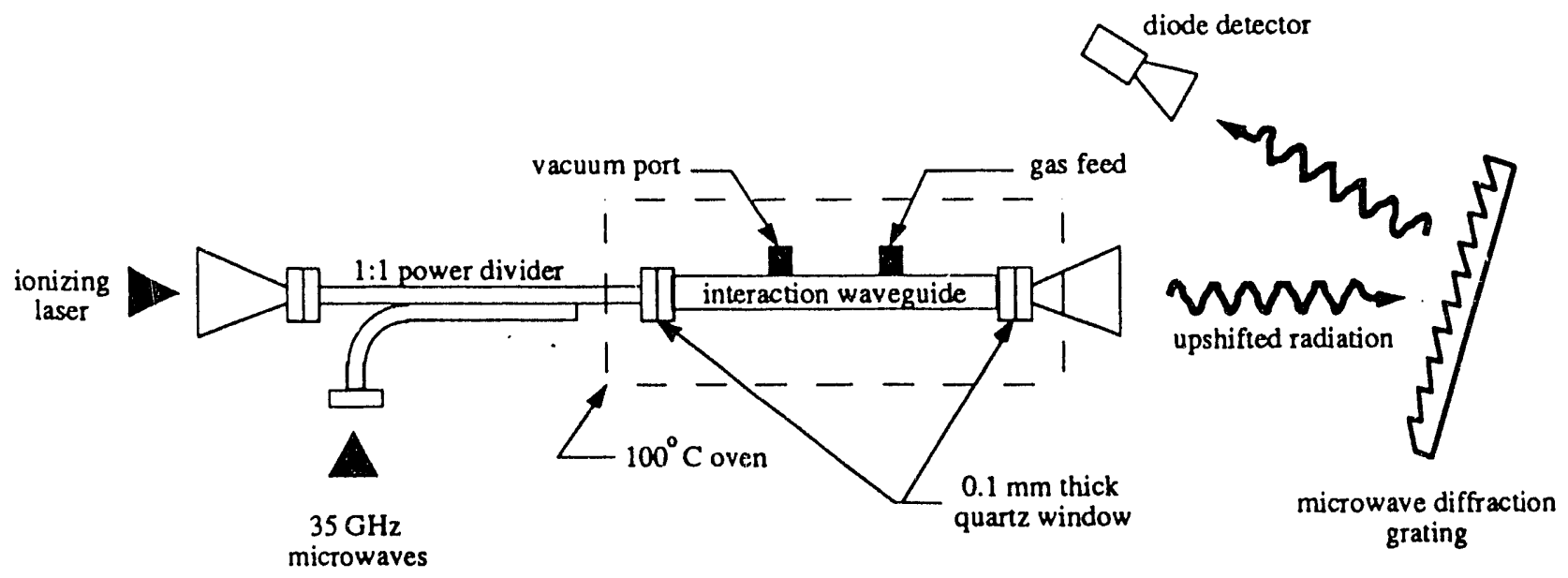

Figure 19: Frequency measurement setup.

For this experiment, we measured two characteristics of the upshifted waves: frequency and temporal width. These measurements were taken with the setup shown in Fig. 19. Using the microwave diffraction grating we were able to measure the frequency spectrum of the upshifted radiation, and from the output signals of our diode detectors we measured the radiation pulsewidth. Figs. 20 and 21 show typical detector signals for an upshifted forward 
and backward wave. Note how broad the backward wave signal is compared to the forward wave (for this particular trace, the backward wave was just below the turnaround point and thus had a long pulsewidth).

Fig. 22 shows a typical frequency spectrum of an upshifted forward wave. If we plot the center frequencies of these spectra for different ionization front densities, we obtain the plot for the upshifted forward wave shown in Fig. 23. The error bars represent the widths (FWIIM) of each frequency spectrum. From this plot we see that the data points are in fair agrecment with the theoretical line. We should note that this plot represents the preliminary results for the diffraction grating frequency measurements of the forward wave. More comprehensive measurements, including backward wave frequency measurements, are planned for the near future.

We do have data from earlier runs which measure the frequency upshift of both the forward and backward wave. This set of frequency data was taken using cutoff waveguides placed in front of the detectors. The procedure for these frequency measurements is as follows: for a given cutoff waveguide we began running the experiment at a low front density so that the frequency of the upshifted radiation would be below the cutoff freciuency of the waveguide. Thus we would see no signal, because the radiation could not propagate through the waveguide to the detector. We then increased the ionization front density until a signal from the detector suddenly appeared. The appearance of this signal would indicate that the wave had been upshifted above the cutoff frequency at that particular density. Using several waveguides having different cutoff frequencies, we were able to create a frequency vs. front density plot in both the forward and backward wave configurations (Fig. 24). The error bars indicate the density range over which the detector signal first appeared. We can see from the plot that the forward wave data points fell nicely on the forward wave theoretical line. However, the backward wave data points seemed to fall closer to the forward wave line rather than the backward wave theory line as we would expect. We'll have more to say about this inconsistency in Sec. 4.5 .

Fig. 25 shows the measured pulsewidths for the forward and backward upshifted waves compared to the theoretical curves. For the forward wave we find good agreement with the theory, particularly at lower density values. We should note that the response time of our diode detectors is $\sim 400 \mathrm{ps}$, and so the data points at high densities are detector limited. For the backward wave we also have good agreement with theory at low densities. At high densities, however, the backward wave data points once again seem to fall closer to the forward wave theoretical curve than the backward wave curve.

\subsection{Experimental Difficulties}

These inconsistencies with the backward wave data may be due to the following difficulties with our experimental setup. First of all, our incident $35 \mathrm{GHz}$ microwaves are not completely unidirectional. When the magnetron launches the microwaves down the waveguide cavity in either the forward or backward configuration, there will always be partial reflections from the $0.1 \mathrm{~mm}$ quartz windows at the ends of the cavity. Thus at the time when the laser 


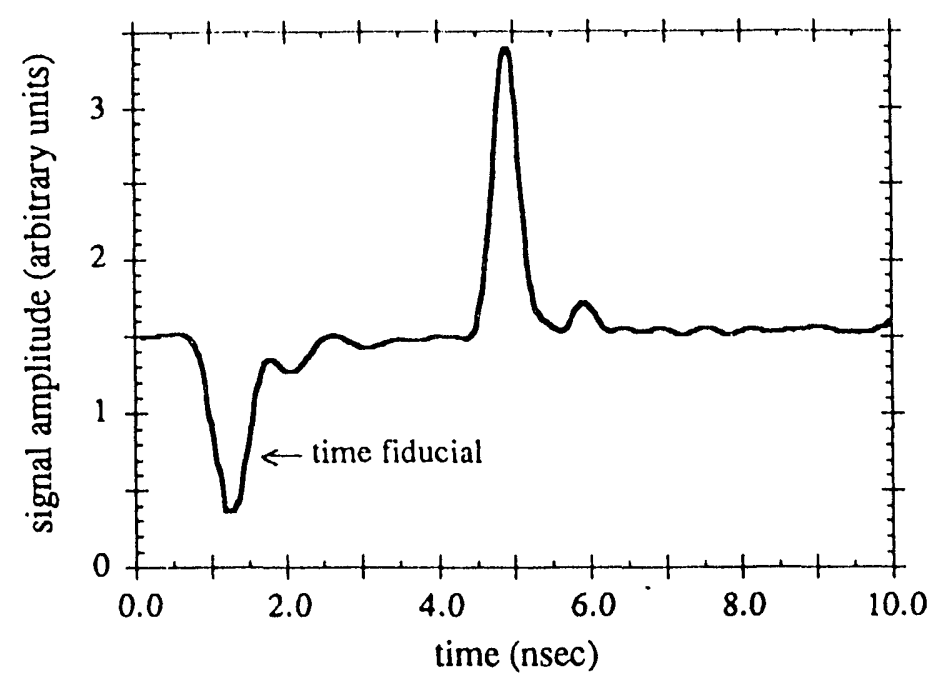

Figure 20: Signal from $173 \mathrm{GHz}$ upshifted forward wave.

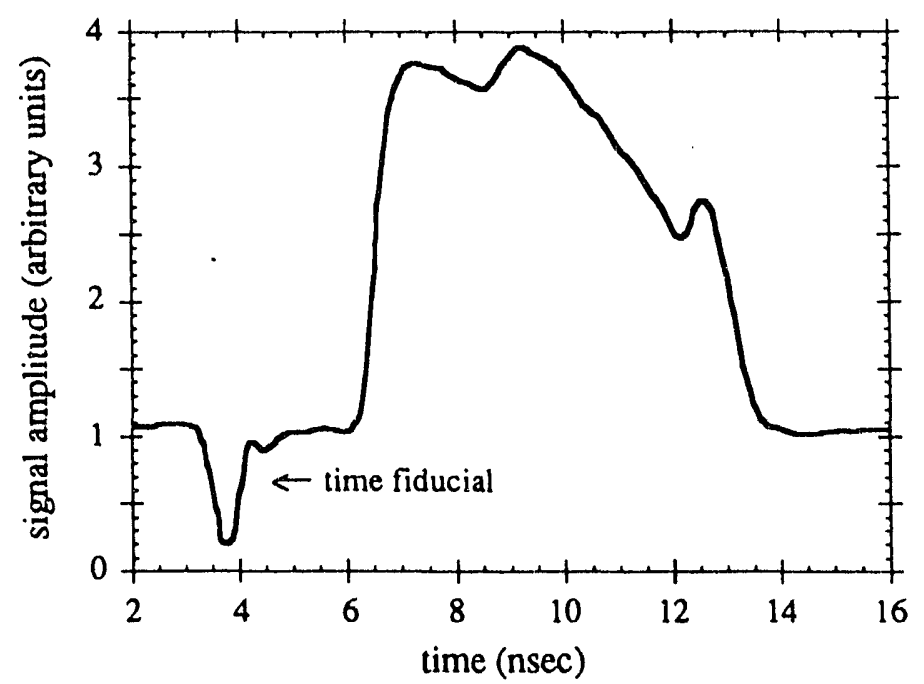

Figure 21: Signal from $40 \mathrm{GHz}$ upshifted backward wave. 


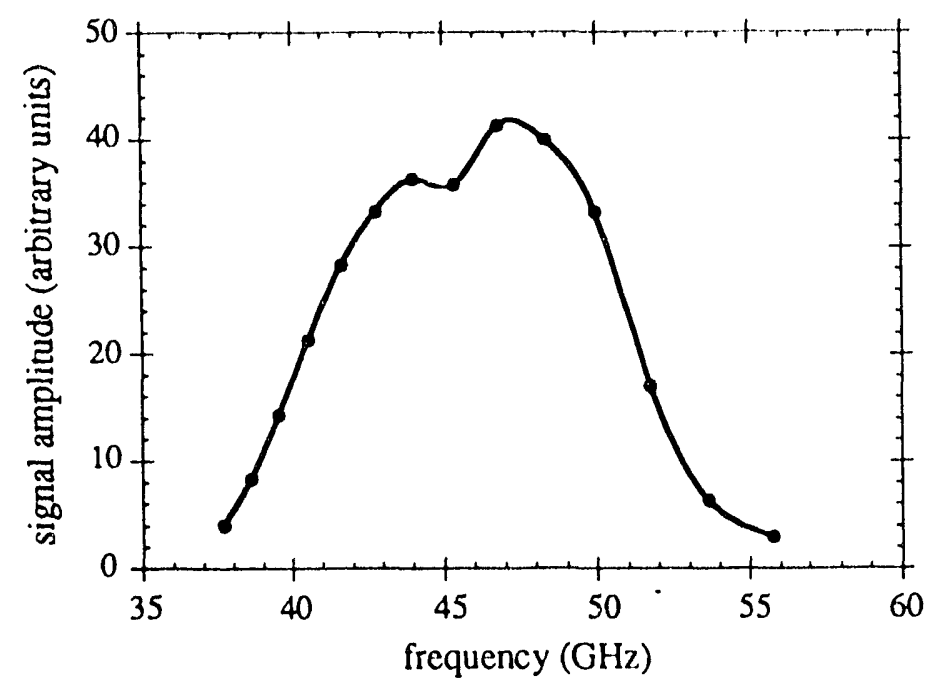

Figure 22: Frequency spectrum for $46 \mathrm{GHz}$ upshifted forward wave.

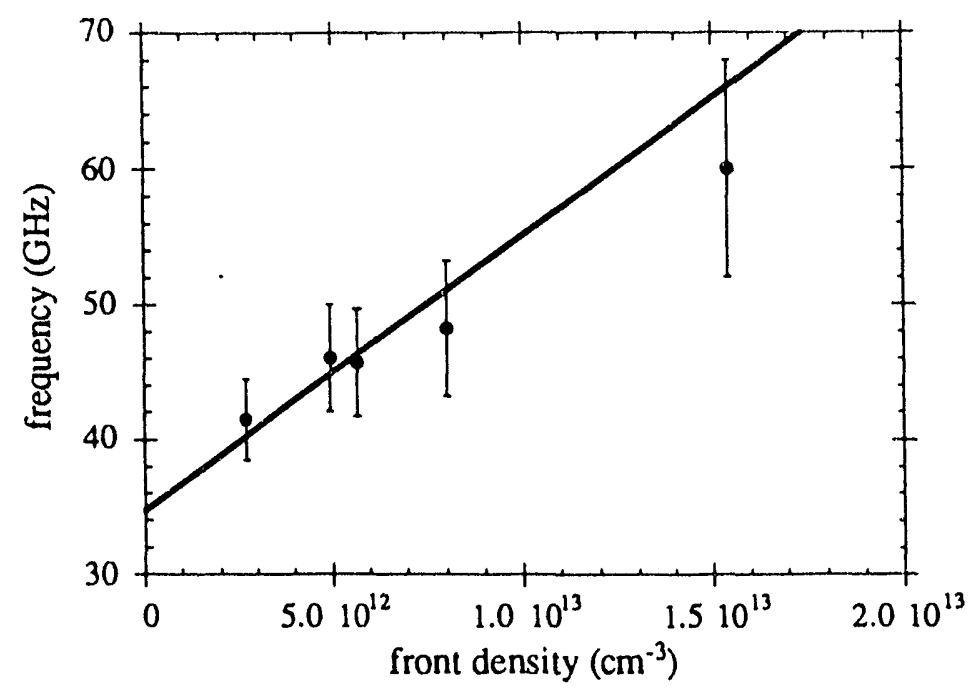

Figure 23: Upshifted frequency data for the forward wave, measured using a microwave diffraction grating. The error bars represent the width of the spectrum for each data point. 


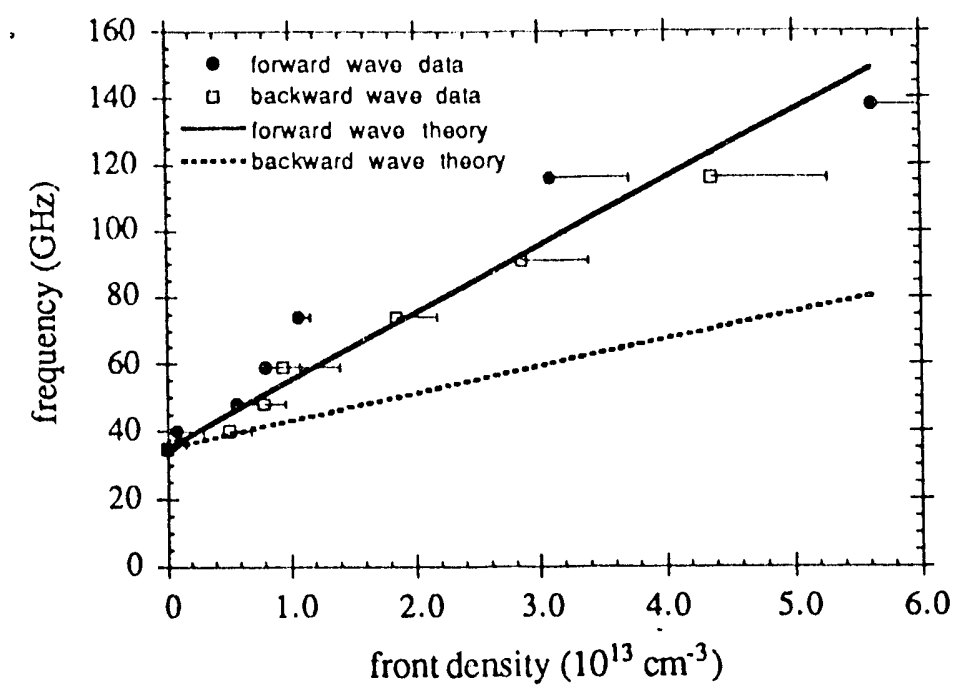

Figure 24: Upshifted frequency data for both the forward and backward waves, taken using cutoff waveguides. The error bars represent the density range over which the detector signal appeared.

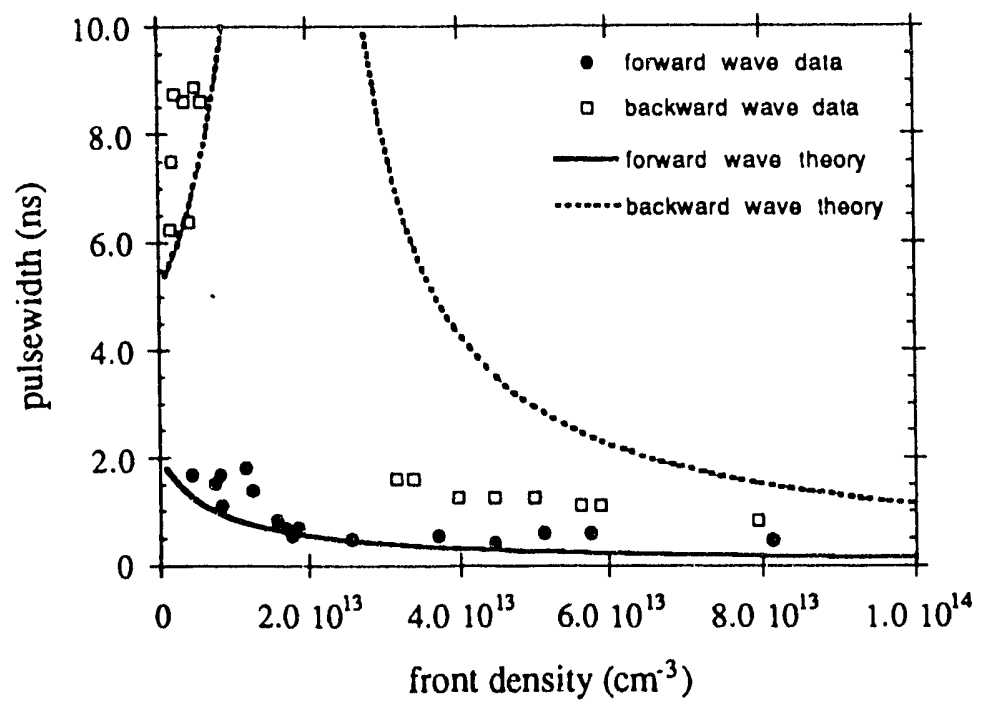

Figure 25: Pulsewidth data for upshifted forward and backward waves. Data points at high densities are detector limited to $0.4 \mathrm{~ns}$. 
pulse is fired, there will be both forward and backward direction microwaves coexisting in the cavity. Because the windows are thin the reflection should be small, but nonetheless we have measured about $10-20 \%$ of the microwave power in the undesired (reflected) direction. Therefore, during the backward wave measurements it is possible that for plasma densitics approaching the critical density for a turnaround, the slower group velocity of the upshifted backward wave causes it to becorne significantly damped in the plasma. (A slower group velocity means the wave spends more time in the plasma before it exits the cavity, thus there is more time for damping effects to take place.) This could mean that above the critical turnaround density we are actually measuring the forward wave (reflected from the quartz window before the laser pulse arrived) even though the experimental setup is in the backward wave cc. figuration. This would explain the behavior of the backward wave data points at high densities. A further complication is this: once the laser pulse has passcd completely through the cavity, there exists a stationary boundary at each quartz window between the newly created plasma inside the chamber and the air outside the chamber. When the upshifted microwave pulse (forward or backward wave) reaches the end of the chamber, part of the pulse will exit through this boundary and the rest will be reflected and travel back through the plasma to the boundary at the other quartz window. At this second boundary the same thing will happen, and so we will observe upshifted radiation coming out both ends of the cavity even though initially the radiation was travelling only in one direction. This fact, coupled with the above non-unidirectionality of the incident microwaves, can make it difficult to distinguish between the forward and backward upshifted wave.

\section{$5 \quad$ Transmitted Wave Turnaround}

Let us now discuss the transmitted wave turnaround for a moving ionization front and ask the question: is this phenomenon a new type of reflection? To answer this, we need to define what we mean by the term "reflection". For an initial, rough definition let us say that a reflection is the reversing of a wave's direction of propagation upon encountering a boundary. For example, when an electromagnetic wave impinges upon a perfect conductor and reverses its direction, this clearly fits our description of a reflection.

What about the case of a wave transmitted into a moving dielectric? As we saw in Sec 3.2, in the laboratory frame under certain conditions $(n \beta>1)$ this transmitted wave will turn around and travel in the opposite direction of the incident wave. Is this a reflection as well? Based on the above definition of a reflection the answer would be "yes", even though the wave has been transmitted through the boundary. This is rather counterintuitive, so let us take a closer look at this particular case and see if our definition requires modification.

To begin, let us transform to the reference frame where the dielectric is stationary. In this frame the transmitted wave always propagates in the same direction as the incident wave, and so there is certainly no reflection here. As we pointed out in the moving dielectric analysis, the apparent transmitted wave turnaround in the lab frame is due to the wave being carried backward by the bulk medium faster than it can propagate forward through it. Thus the turnaround in this case is simply a frame of reference effect, because in the the 
rest frame of the medium through which the wave propagates there is no such turnaround. Therefore we can qualify our definition to state that a refection is the reversing of a wave's direction of propagation in the rest frame of the material through which the wave is traveling. In the framework of this definition, then, the turnaround of a wave transmitted into a moving dielectric is not a reflection.

Finally, lets look at the case of the electromagnetic wave cncountering an ionization front moving through a stationary plasma. In the laboratory frame, the boundary moves but the medium through which the waves travel (the plasma and neutral gas) remain stationary. This implies that the laboratory frame is the preferred frame of reference for this problem. We showed in Sec 3.4 that for a moving ionization front the transmitted wave turns around for front denstities above a particular critical density $\left(\omega_{p}^{2}>4 \omega_{0}^{2}\right.$ in free space).

Does this constitute a reflection? If we transform to the rest frame of the ionization front nothing special seems to happen -- the incoming wave is simply transmitted through the boundary at a lower group velocity. Thus if we were to change our definition to state that a reflection is the reversing of a wave's direction in the reference frame of the boundary, then the transmitted wave turnaround would not qualify as a reflection. However, this frame is not the preferred reference frame because the medium of plasma and neutral gas particles through which the wave travels is not stationary, but instead is moving with velocity $\beta$.

The answer to this question, then, depends on our definition of the term "reflection". If we define it to be the reversing of a wave's direction in the rest frame of the boundary, the transmitted wave turnaround is certainly not a reflection. However, if we define a reflection to be the reversing of a wave's direction in the rest frame of the medium through which the wave is propagating, then the transmitted wave turnaround does fit the criteria, and thus can be considered a new type of electromagnetic reflection.

\section{$6 \quad$ Future Experiments}

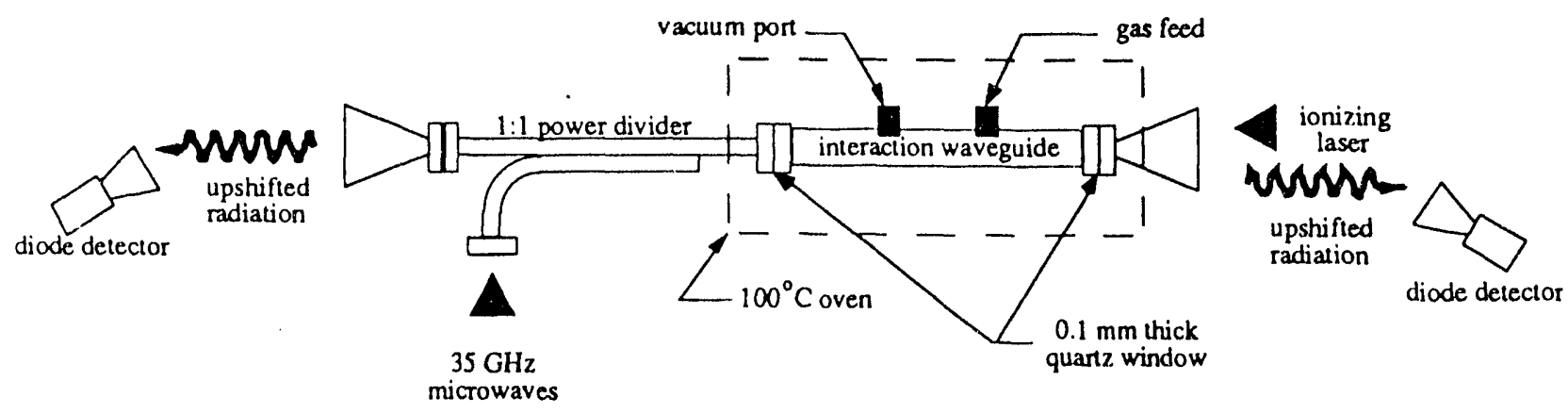

Figure 26: Setup for time resolvable upshift experiment.

Obviously, we would like to verify this transmitted wave turnaround experimentally. Unfortunately, the difficulties in the experiment described earlier have made us unable to conclusively record this phenomenon at present. One of the problems mentioned earlier is our 
inability to distinguish in which direction the upshifted wave was initially traveling. In our next experiment, we hope to remedy this by setting up detectors at equal distances from the chamber in both directions (Fig. 26). By looking at the timing of the detector signals when an upshifted wave is created in the backward wave configuration, we should be able to determine which detector recorded the original upshifted wave (the carlier signal), and which one detected a reflection of the wave from the chamber window (the later signal). When we compare the results of this timing expcriment for low front densitics $\left(n<2 \times 10^{13} \mathrm{~cm}^{-3}\right)$ to the results at high densities $\left(n>2 \times 10^{13} \mathrm{~cm}^{-3}\right)$, we should observe that the initial direction of propagation has changed and thus the wave has turned around. This technique may demonstrate the backward wave turnaround provided that there is not too much damping. If the turned-around backward wave is damped significantly in the plasma, we may have difficulty distinguishing it frorn the residual forward wave in the chamber because both waves will have similar amplitudes and will be propagating in the same direction.

Thus our next set of experiments will employ careful amplitude and timing measurements in an attempt to overcome the above difficulties. If successful, we hope to find evidence of this rather interesting type of "reflection": the transmitted wave turnaround.

\section{References}

[1] V. I. Semenova, Sov. Radiophys. 10, 599 (1967).

[2] M. Lampe, E. Ott, and J. H. Walker, Phys. Fluids 21, 42 (1978).

[3] W.B. Mori, Phys. Rev. A 44, 5118 (1991).

[4] R. L. Savage, Jr., C. Joshi, and W. B. Mori, Phys. Rev. Lett. 68, 946 (1992).

[5] J. D. Jackson, Classical Electrodynamics, Second Edition. (Wiley, New York,1975).

[6] D. J. Griffiths, Introduction to Electrodynamics, (Prentice Hall, New Jersey, 1981) 

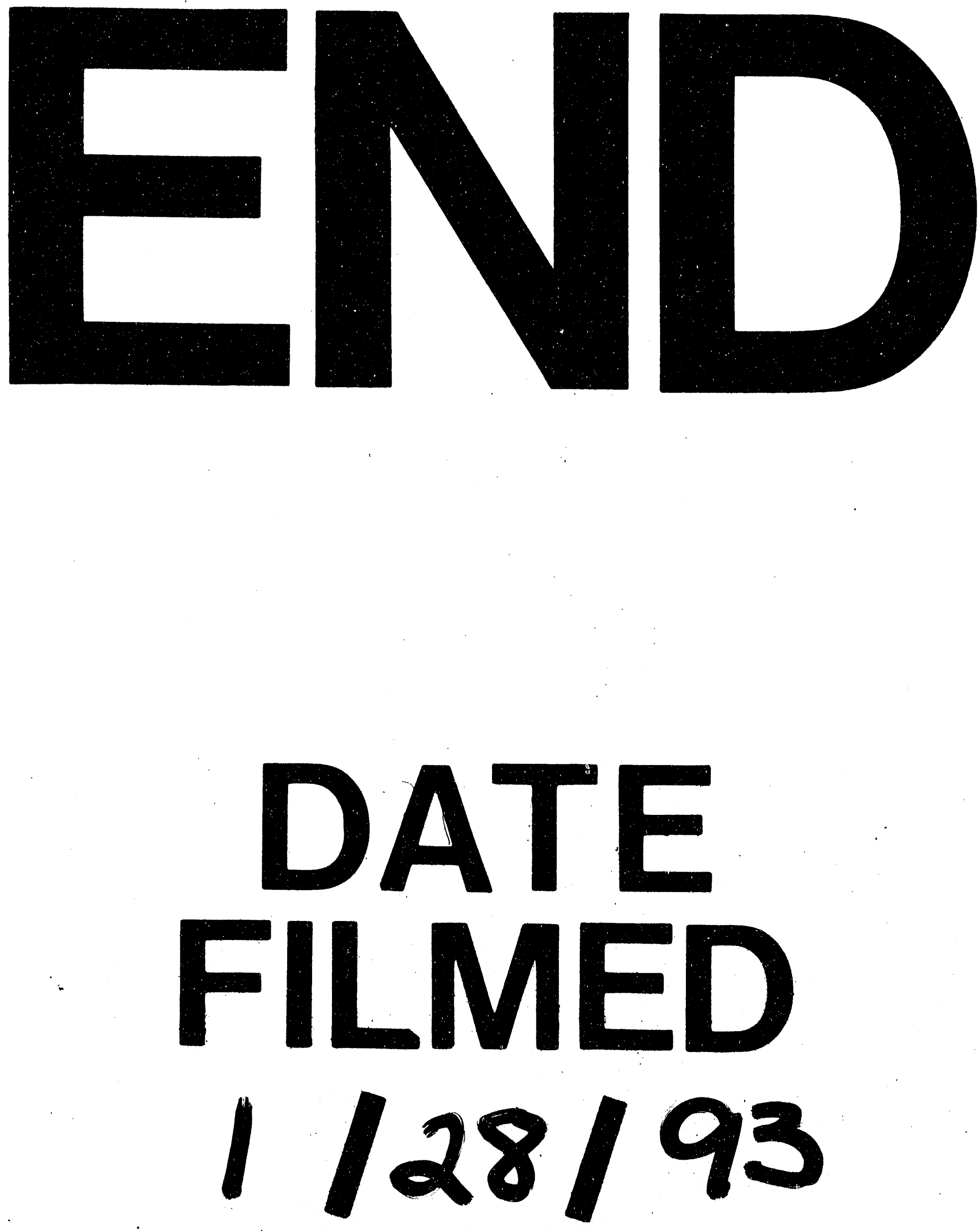
